\title{
A degrading Bouc-Wen model for the hysteresis of reinforced concrete structural elements
}

\author{
Matteo Pelliciari ${ }^{\mathrm{a}, \mathrm{b}}$, Bruno Briseghella ${ }^{\mathrm{a}}$, Francesco Tondolo ${ }^{\mathrm{c}}$, Luigi Veneziano ${ }^{\mathrm{b}}$, Camillo Nuti $^{\mathrm{d}}$, \\ Rita Greco $^{\mathrm{e}}$, Davide Lavorato ${ }^{\mathrm{d}}$ and Angelo Marcello Tarantino ${ }^{\mathrm{b}}$ \\ ${ }^{a}$ College of Civil Engineering, Fuzhou University, Fuzhou, China; ${ }^{b}$ DIEF, University of Modena and \\ Reggio Emilia, Modena, Italy; ${ }^{\mathrm{c}}$ DISEG, Polytechnic University of Turin, Turin, Italy; ${ }^{\mathrm{d}}$ Department of

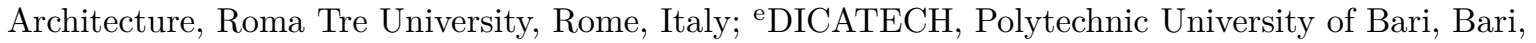 \\ Italy.
}

\begin{abstract}
ARTICLE HISTORY
Compiled September 26, 2019

ABSTRACT

This paper presents a smooth hysteresis model for reinforced concrete structural elements based on the differential equation of the Bouc-Wen model. Stiffness degradation and strength degradation are defined by introducing a damage index that includes both dissipated energy and maximum displacement. The pinching effect acts directly on the stiffness of the system and is controlled by an activation energy. The degrading functions are connected to the actual processes with which the damage occurs, thereby giving each parameter a physical meaning. The simple formulation of the model allows a straightforward identification of the best fitting parameters and an easy interpretation of the results. Applications to the cyclic behaviour of reinforced concrete structural elements demonstrate that the model is well capable of describing complex hysteretic behaviours involving degradation and pinching effects.
\end{abstract}

\section{KEYWORDS}

Mathematical models; nonlinear analysis; concrete, reinforced; damage; identification; structural response; bridges, piers.

\section{Introduction}

The hysteresis phenomenon is observed in various mechanical systems. For instance, engineering structures often exhibit hysteretic behaviour under severe cyclic loads caused by earthquakes (Roufaiel \& Meyer, 1987). Repeated cyclic deformations frequently cause a deterioration of the characteristics of reinforced concrete (RC) mechanical systems. In fact, building structures may experience opening and closing of cracks, post-yielding and buckling of metallic elements (Kashani, Salami, Goda, \& Alexander, 2018), strength and stiffness deterioration, and other local inelastic behaviours (Sivaselvan \& Reinhorn, 2000). All these phenomena contribute to the structural damage. An accurate prediction of the behaviour resulting from a cyclic loading must take into account the damage of the structure. Therefore, constitutive models capable of representing nonlinear deterioration are required.

The evaluation of the seismic damage of structures needs, firstly, a definition of a suitable measure for the damage itself. Most of the damage indicators available in literature involve two quantities: the energy dissipated during the inelastic cycles of hysteresis and the displacement ductility, defined as the ratio between maximum displacement and yielding displacement. For instance, Park and Ang (1985), Kunnath, Reinhorn, and Park (1990), Banon and Veneziano (1982) and Singhal and Kiremidjian (1996) considered both ductility and dissipated energy to assess structural damage phenomena. Greco and Marano $(2015 ; 2006)$ employed a damage in- 
dex involving displacement ductility and hysteretic energy to perform a stochastic analysis of the cumulative damage in structural systems subjected to ground motions. Elenas (2000) and Bassam, Iranmanesh, and Ansari (2011) performed, respectively, an evaluation of the damage of reinforced concrete bridges and reinforced concrete frame structures based on ductility and energy dissipated by the system. Consequently, mathematical models able to represent hysteretic cycles with degradation should involve both displacement ductility and dissipated energy.

The simplest models to describe the time-dependent hysteretic behaviour are the bilinear and trilinear models (see, e.g, Liu, Zordan, Zhang, \& Briseghella, 2015; Markou \& Manolis, 2016; Ray, Sarlis, Reinhorn, \& Constantinou, 2013). However, the hysteresis actually happens as a smooth phenomenon. Thus, smooth hysteresis models based on differential equations have been proposed. One of the most widely established is the Bouc-Wen model (Bouc, 1971; Wen, 1976).

The Bouc-Wen model has been employed for many engineering applications, such as RC beams and columns (Kunnath, Mander, \& Fang, 1997; Lee \& Han, 2018; Loh, Mao, Huang, \& Pan, 2011; Wang, Lu, \& Ye, 2007), piezoelectric actuators (Zhu \& Rui, 2016), seismic isolators (Domaneschi, 2012; Ismail, Rodellar, \& Ikhouane, 2010; Manzoori \& Toopchi-Nezhad, 2017), steel connections (Charalampakis \& Dimou, 2010; Kim \& Lee, 2019), and others. It has been demonstrated that the Bouc-Wen model is well-capable of predicting the hysteresis of mechanical systems. Nevertheless, its mathematical formulation does not include degradation phenomena. Indeed, numerous authors extended its governing equations in order to consider the degradation of the system (see, e.g., Baber \& Noori, 1985; Erlicher \& Bursi, 2008; Kottari, Charalampakis, \& Koumousis, 2014; Pelliciari et al., 2018). In particular, the Bouc-Wen-Baber-Noori (BWBN) model (Baber \& Noori, 1985) has been considerably successful.

The BWBN model is an extension of the Bouc-Wen model, developed through the incorporation of functions that describe degradation and pinching. However, as pointed out by Marano et al. (2017), the formulation of the BWBN model is complicated and not all the parameters involved have a direct physical meaning. Furthermore, degradation and pinching are described only on the basis of the dissipated energy.

In this work, a smooth hysteresis model for degrading RC structural elements is presented. The model is based on the differential equation of the Bouc-Wen model, to which appropriate degrading functions are added in order to depict the cyclic damage. Stiffness degradation and strength degradation are defined through a damage index that involves both maximum displacement and dissipated energy. Moreover, the pinching effect is depicted along the same lines of the simple formulation proposed by Pelliciari et al. (2018), incorporating modifications that correct its physical deficiencies.

The mathematical description of the degradation of the system is based on the physical processes with which the structural damage takes place. Thereby, each parameter of the proposed model is physically based and related to a certain damage phenomenon, allowing an easy control and interpretation of the results. The formulation of the present model is straightforward and oriented towards practical engineering applications. Indeed, the purpose of this study is to provide a simple and reliable model able to predict the hysteresis of RC structural elements with pinching and degradation, including at once a physically-based description of the damage.

The paper is organized as follows. In Section 2, an outline of the Bouc-Wen model is provided. The damage index is then introduced and the functions that account for the mechanical degradation of the system are described, including the pinching effect. Section 3 provides an overview of the parameter optimization strategy, which is based on a genetic algorithm (GA). Consequently, in Section 4, the effectiveness of the proposed model is verified by its application to the cyclic behaviour of a circular RC bridge pier and two rectangular RC columns. Such structural systems exhibit complex cyclic behaviours, involving significant damage. Finally, conclusions are drawn in Section 5. 


\section{Hysteresis model for degrading systems}

In this section, the Bouc-Wen model of hysteresis is briefly introduced. At the same time, each improvement that allows to account for degrading phenomena is discussed.

\subsection{Preliminaries on the Bouc-Wen model}

The equation of motion for a single-degree-of-freedom system is

$$
m \ddot{x}+c \dot{x}+F_{s}[x(t), z(t), t]=F(t),
$$

where $x$ is the relative displacement of the mass of the system $m$ with respect to the ground, $c$ is the linear viscous damping coefficient, $F_{s}[x(t), z(t), t]$ is the non-damping restoring force, $z(t)$ is the hysteresis displacement and $F(t)$ is the external excitation. The overdots indicate the derivative with respect to the time, thus $\dot{x}$ and $\ddot{x}$ represent velocity and acceleration, respectively.

The Bouc-Wen model gives the following expression for the restoring force:

$$
F_{s}[x(t), z(t), t]=\alpha k x(t)+(1-\alpha) k z(t),
$$

where $k$ is the elastic stiffness of the system and $\alpha$ is the ratio between the final tangent stiffness $k_{f}$ and the elastic stiffness $(0 \leq \alpha \leq 1)$. Equation $(2)$ is composed of two contributions: the linear elastic component $\alpha k x(t)$ and the hysteresis component $(1-\alpha) k z(t)$, which depends on the past history of stresses and strains.

The hysteresis displacement $z(t)$ is given by the differential equation

$$
\dot{z}(t)=A \dot{x}(t)-\left[\beta|\dot{x}(t)||z(t)|^{n-1} z(t)+\gamma \dot{x}(t)|z(t)|^{n}\right],
$$

with the initial condition $z(0)=0$. The parameters $\beta, \gamma$, and $n$ control the shape of the hysteresis cycles, while $A$ determines the tangent stiffness. As is well-known in literature (Charalampakis \& Koumousis, 2008b; Ma, Zhang, Bockstedte, Foliente, \& Paevere, 2004), the aforementioned parameters are redundant. Thus, $A$ is commonly set to unity in order to eliminate this redundancy. However, since in this work the parameter $A$ will be used in the mathematical developments for the description of the degradation, this position will be performed later (see Section 2.3). Note also that Ismail, Ikhouane, and Rodellar (2009) gave the following conditions for the Bouc-Wen model parameters in order to satisfy the thermodynamic admissibility, the accordance with Drucker and Il'iushin stability postulates and the uniqueness of the solution:

$$
\begin{aligned}
& \beta>0, \quad-\beta \leq \gamma \leq \beta, \\
& \beta+\gamma>0, \quad \gamma-\beta \leq 0, \\
& n \geq 1
\end{aligned}
$$

The hysteresis energy dissipated by the system is defined as the area under the hysteresis restoring force $F^{h}[z(t), t]=(1-\alpha) k z(t)$ along the total displacement $x(t)$. The hysteresis energy can be normalized with respect to the mass, as follows (Foliente, 1995; Ortiz, Alvarez, \& BedoyaRuíZ, 2013):

$$
\epsilon(t)=\int_{x(0)}^{x(t)} \frac{F^{h}[z(t), t]}{m} d x=(1-\alpha) \omega_{0}^{2} \int_{0}^{t} z(\tau) \dot{x}(\tau) d \tau .
$$

Some authors found out that for many structural systems the two parameters $\beta$ and $\gamma$ are related to each other. For instance, Sues, Mau, and Wen (1988) suggested that $\beta=\gamma$ for steel structures and $\beta=-3 \gamma$ for RC structures, whereas Sengupta and Li (2013) applied a BoucWen model to RC beam-column joints, obtaining $\beta=-5 \gamma$. Ye and Wang (2007) estimated the 
Bouc-Wen model parameters considering both the cases of softening and hardening behaviour. The results showed that for a system with softening $\beta \cong 2 \gamma$, while for a system with hardening $\beta \cong-\gamma$. In view of the above, it appears reasonable to establish a linear proportionality between the parameters $\beta$ and $\gamma$

$$
\gamma=\eta_{0} \beta
$$

Given this position, the conditions (4) reduce to

$$
\beta>0, \quad-1<\eta_{0} \leq 1, \quad n \geq 1 .
$$

Note that, with the position (6), the constraints on the variability of the parameters are now decoupled, as expressed by Equation (7). This simplifies the mathematical formulation of the model and represents a great advantage during the identification of the optimal parameters.

Substituting Equation (6) into Equation (3), the differential equation for the hysteretic displacement becomes

$$
\dot{z}(t)=A \dot{x}(t)-\beta\left[|\dot{x}(t)||z(t)|^{n-1} z(t)+\eta_{0} \dot{x}(t)|z(t)|^{n}\right] .
$$

\subsection{Damage index}

As already pointed out, the most popular damage indicators involve both hysteretic dissipated energy and maximum displacement (Park \& Ang, 1985). Hence, in this work the authors propose a model that connects the degradation phenomena to the following dimensionless damage index:

$$
d_{i}(t)=\rho_{\epsilon} \frac{\epsilon(t)}{f_{y} x_{u}}+\rho_{x} \frac{\left|x_{\max }(t)\right|}{x_{u}},
$$

where $x_{u}$ is the ultimate displacement under a monotonic loading, $f_{y}=F_{y} / m$ is the yielding force normalized with respect to the mass and $x_{\max }(t)$ is the maximum displacement of the system until the time $t$. Equation (9) brings two new parameters into the hysteresis model: $\rho_{\epsilon}$ and $\rho_{x}$. Their purpose is to weigh the influence of the degradation due to the energy dissipation and the maximum displacement, respectively.

Note that the magnitude of both $\epsilon(t)$ and $x_{\max }(t)$ is not known a priori. On the contrary, it depends on the excitation to which the mechanical system is subjected. Therefore, it is not possible to normalize Equation (9) in a way that $\rho_{\epsilon}$ and $\rho_{x}$ vary in the range between 0 and 1.

\subsection{Stiffness and strength degradation}

For systems with softening behaviour, the following derivatives can be computed (Baber \& Wen, 1980):

$$
\begin{aligned}
& k_{i}=\left(\frac{\partial F}{\partial x}\right)_{z=0}=\alpha k+(1+\alpha) k A, \\
& k_{f}=\left(\frac{\partial F}{\partial x}\right)_{z=z_{u}}=\alpha k,
\end{aligned}
$$

where the ultimate value of the hysteretic displacement, $z_{u}$, is expressed by

$$
z_{u}=\left[\frac{A}{\beta_{0}\left(1+\eta_{0}\right)}\right]^{\frac{1}{n}} .
$$




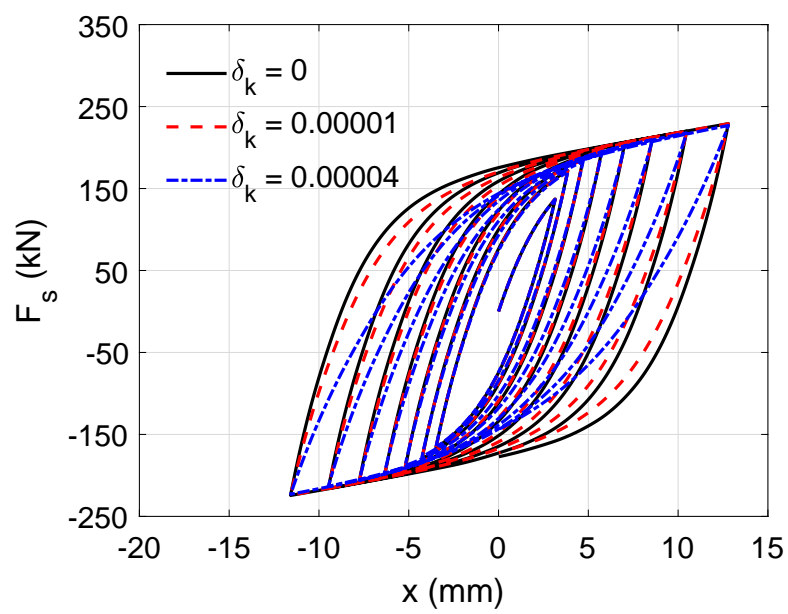

Figure 1. Effect of the parameter $\delta_{k}$ on the hysteresis cycles (stiffness degradation).

The ultimate hysteretic restoring force component can be thus defined as (Cunha, 1994)

$$
F_{h}^{u}=(1-\alpha) k z_{u}=(1-\alpha) k\left[\frac{A}{\beta_{0}\left(1+\eta_{0}\right)}\right]^{\frac{1}{n}} .
$$

The stiffness degradation can be introduced into the model by expressing $k_{i}$ as a function that degrades with the damage index $d_{i}(t)$. Nevertheless, the elastic stiffness $k$ should not be subjected to any degradation. Thus, looking at Equation (10), one can conclude that the degradation of the stiffness must be entirely included into the parameter $A$, by substituting this parameter with a function of $d_{i}(t)$.

In order to decouple stiffness and strength degradation, the ultimate hysteretic restoring force component must remain constant while the degradation of the function $A\left(d_{i}\right)$ occurs. Therefore, the parameter $\beta_{0}$ in Equation (13) is replaced by a function $\beta_{k}\left(d_{i}\right)$, in such a way that the following relation holds:

$$
F_{h}^{u}=(1-\alpha) k\left[\frac{A\left(d_{i}\right)}{\beta_{k}\left(d_{i}\right)\left(1+\eta_{0}\right)}\right]^{\frac{1}{n}}=\text { const. }
$$

It is often assumed that the damage of a structural system evolves with the dissipation of energy following a negative exponential law (see, among the others, Bažant, Pan, \& PijaudierCabot, 1987; Lanzoni \& Tarantino, 2014, 2015, 2016; Mazars, 1986; Miehe, 1995; Tarantino, 2014). Thus, the following form for the function $A\left(d_{i}\right)$ is adopted:

$$
A\left(d_{i}\right)=e^{-\delta_{k} d_{i}(t)}
$$

where $\delta_{k}$ is a parameter that controls the amount of stiffness degradation. Obviously, for $\delta_{k}=0$, the model does not exhibit stiffness degradation. Note that the redundancy of the Bouc-Wen model described in Section 2.1 is now removed by turning the parameter $A$ into the function $A\left(d_{i}\right)$, where the only unknown parameter is $\delta_{k}$. At this point, given (15), Equation (14) is satisfied if

$$
\beta_{k}\left(d_{i}\right)=\beta_{0} e^{-\delta_{k} d_{i}(t)}
$$

This procedure allows incorporating the whole description of the stiffness degradation into the function $\beta_{k}\left(d_{i}\right)$.

Figure 1 shows the effect of variations of $\delta_{k}$ on the hysteresis cycles. The higher the value of 


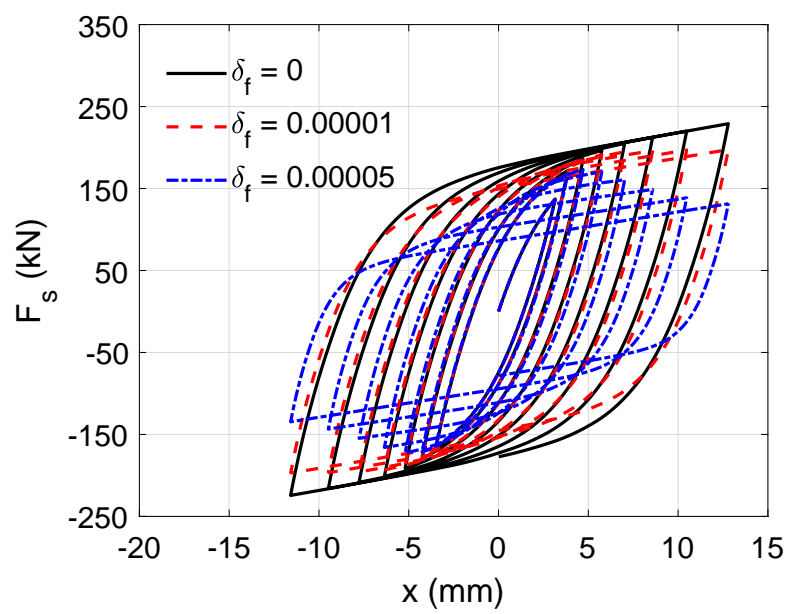

Figure 2. Effect of the parameter $\delta_{f}$ on the hysteresis cycles (strength degradation).

$\delta_{k}$, the more the degradation of the stiffness is pronounced. It is worth noting that the maximum value of the force acting on the system is not influenced by the stiffness degradation.

In the following, the strength degradation is described separately from the stiffness degradation and the two formulations will be combined at the end of the present section. Similarly to what has been done above, the degradation of the strength is introduced in a way that it is decoupled from the degradation of the stiffness. This is done by defining the ultimate hysteretic restoring force $F_{h}^{u}$ as a degrading function of $d_{i}(t)$. Hence, the initial stiffness $k_{i}$ must remain constant. This means that, looking at Equation (10), the parameter $A$ must be constant. Therefore, the degradation of the strength is introduced by replacing $\beta_{0}$ with $\beta_{f}\left(d_{i}\right)$ into Equation (13), with $\beta_{f}\left(d_{i}\right)$ defined by the following exponential law:

$$
\beta_{f}\left(d_{i}\right)=\beta_{0} e^{n \delta_{f} d_{i}(t)},
$$

where $\delta_{f}$ is a parameter that controls the amount of strength degradation. Note that for $\delta_{f}=0$ there is no strength degradation.

Substituting Equation (17) into Equation (13), the following relation is obtained:

$$
F_{h}^{u}=(1-\alpha) k\left[\frac{A}{\beta_{f}\left(d_{i}\right)\left(1+\eta_{0}\right)}\right]^{\frac{1}{n}}=(1-\alpha) k\left[\frac{A}{\beta_{0}\left(1+\eta_{0}\right)}\right]^{\frac{1}{n}} e^{-\delta_{f} d_{i}(t)},
$$

which states that the degradation of the strength follows a negative exponential law with the damage index $d_{i}(t)$. Note that when strength and stiffness degradation are combined, the parameter $A$ is replaced by the function $A\left(d_{i}\right)$ expressed by Equation (15), where the redundancy of the Bouc-Wen model has already been removed. In addition, it should be pointed out that the description of the strength degradation is entirely included in the function $\beta_{f}\left(d_{i}\right)$, in analogy with $\beta_{k}\left(d_{i}\right)$ for the stiffness degradation.

Figure 2 shows the effect of variations of $\delta_{f}$ on the hysteresis cycles. Increasing the value of $\delta_{f}$, the degradation of the strength increases. It is noted that the initial slope of the cycles, which represents the initial stiffness $k_{i}$, remains unchanged.

Stiffness and strength degradation have been described above by establishing proper degrading functions of the damage index. However, as already pointed out by Pelliciari et al. (2018), the rate of degradation of the stiffness of a system might not always maintain the same proportionality to the energy (in this case to the damage index). For instance, the degradation rate could be stronger at the beginning of the inelastic phenomena. Then, when the system is severely damaged, its proportionality to the damage index could become less. Thus, the increasing of stiffness degradation rate is here controlled introducing a negative exponential function $p_{k}\left(d_{i}\right)$ 


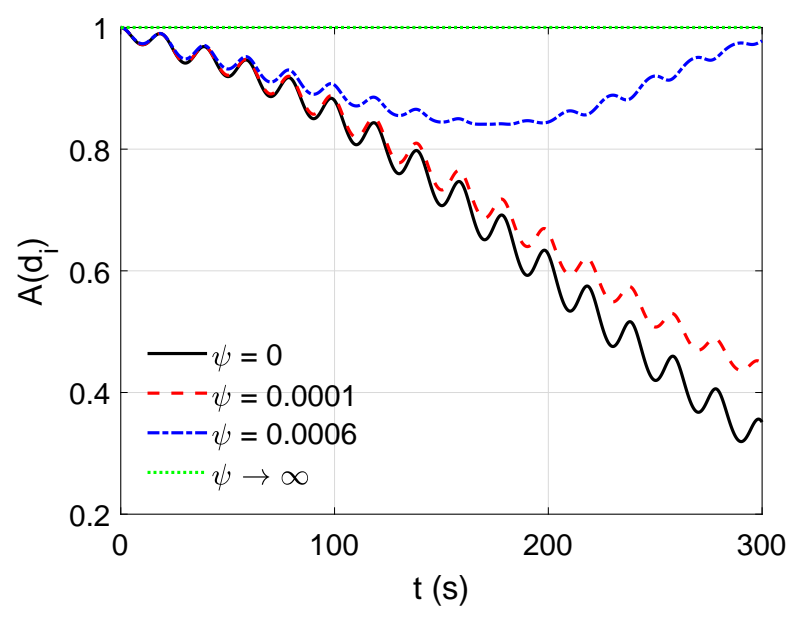

Figure 3. Effect of the parameter $\psi$ on the function $A\left(d_{i}\right)$, which defines the stiffness degradation.

into Equations (15) and (16):

$$
\begin{aligned}
& A\left(d_{i}\right)=e^{-\delta_{k} d_{i}(t) p_{k}\left(d_{i}\right)}, \\
& \beta_{k}\left(d_{i}\right)=\beta_{0} e^{-\delta_{k} d_{i}(t) p_{k}\left(d_{i}\right)}, \\
& p_{k}\left(d_{i}\right)=e^{-\psi d_{i}(t)}
\end{aligned}
$$

where $\psi$ is a parameter that controls the rising of stiffness degradation. Specifically, for $\psi=0$ the stiffness degradation rate follows the increasing of $d_{i}(t)$ through the exponential law expressed by Equation (15). Instead, as $\psi$ increases, the stiffness degradation rate decreases its proportionality to $d_{i}(t)$ as the event proceeds. The limit situation is $\psi \rightarrow \infty$, for which $A\left(d_{i}\right) \rightarrow 1$. Therefore, in this last case, no stiffness degradation occurs. In order to clarify the role of $\psi$, its effect on the hysteresis cycles is displayed in Figure 3.

At this point, the effects of both strength and stiffness degradation are combined by defining a single function that includes both degradation phenomena

$$
\beta\left(d_{i}\right)=\beta_{k}\left(d_{i}\right) \beta_{f}\left(d_{i}\right)=\beta_{0} e^{-\left[\delta_{k} p_{k}\left(d_{i}\right)-n \delta_{f}\right] d_{i}(t)},
$$

and the differential equation for the degrading hysteresis model assumes the form

$$
\begin{aligned}
\dot{z} & =A\left(d_{i}\right) \dot{x}-\beta\left(d_{i}\right)\left(|\dot{x}||z|^{n-1} z+\eta_{0} \dot{x}|z|^{n}\right) \\
& =e^{-\delta_{k} d_{i} p_{k}\left(d_{i}\right)} \dot{x}-\beta_{0} e^{-\left[\delta_{k} p_{k}\left(d_{i}\right)-n \delta_{f}\right] d_{i}}\left(|\dot{x}||z|^{n-1} z+\eta_{0} \dot{x}|z|^{n}\right),
\end{aligned}
$$

where the function $p_{k}\left(d_{i}\right)$ is expressed by Equation (21). For the sake of clarity, the dependence on the time $t$ has been omitted.

Equation (23) governs the hysteresis model including the degradation of stiffness (controlled by the parameters $\delta_{k}$ and $\psi$ ) and the degradation of strength (controlled by the parameter $\delta_{f}$ ). In the following, the pinching effect will be introduced.

\subsection{Pinching effect}

A phenomenological and straightforward way of simulating the pinching phenomenon is to consider it as a degradation process that acts on the stiffness of the system. More precisely, it can be represented as a reduction of the stiffness that occurs in a central range of the force-displacement curve, followed by an increase in the outside regions. It goes without saying that this is not a strict 


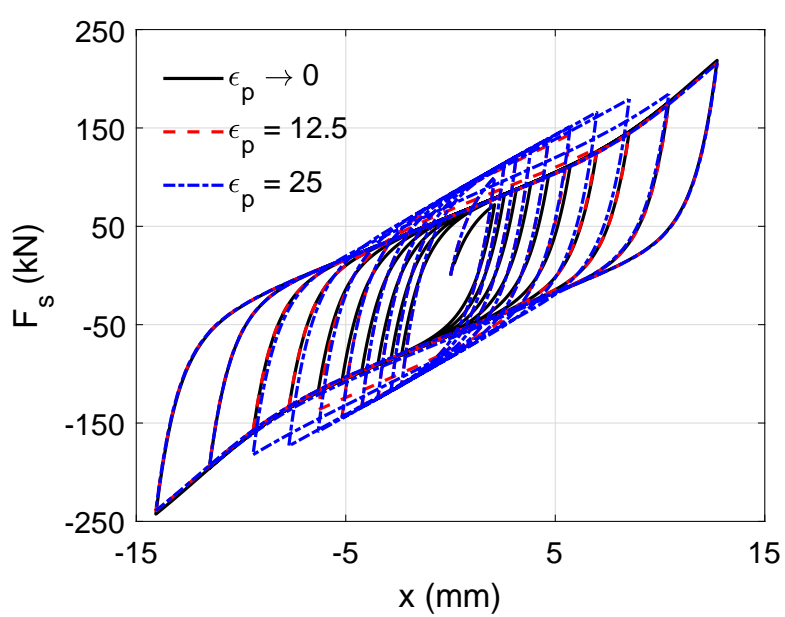

(a) Effect of $\epsilon_{p}$ on the hysteresis cycles.

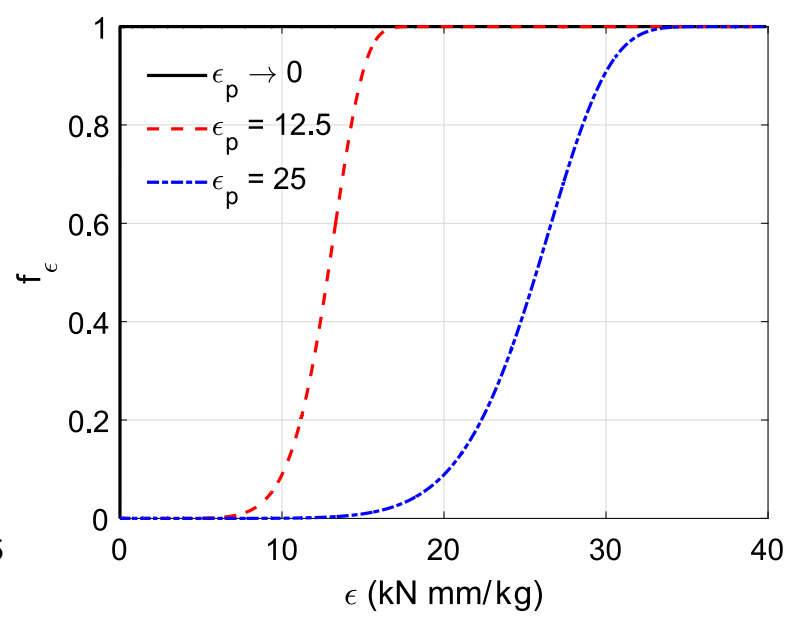

(b) Shape of the exponential function $f_{\epsilon}(\epsilon)$.

Figure 4. Activation of the pinching effect through the parameter $\epsilon_{p}$.

definition, being the nature of pinching accidental and extremely complex. Nevertheless, this definition was employed by Marano et al. (2017) and Pelliciari et al. (2018), obtaining promising results from the application on RC bridge piers.

In the aforementioned works, the authors described this effect through a Gaussian function. However, in their model, this effect starts at the beginning of the event. Hence, the pinching affects every hysteresis cycle, including the very first ones. This is obviously not consistent with the reality, or at least it does not happen for every kind of structural elements and for every displacement history. Therefore, in this work, the same analytical description of the pinching will be adopted, but with the introduction of a new parameter that acts as an activation energy for the pinching effect.

The pinching function is defined as a normalized Gaussian function with mean equal to zero

$$
g_{p}(x)=e^{-\frac{1}{2}\left(\frac{x}{\sigma}\right)^{u}} .
$$

The higher the value of the exponent $u$, the steeper the stiffness change, while the standard deviation $\sigma$ controls the width of the Gaussian bell (see Pelliciari et al., 2018, for a more detailed description). The function $g_{p}(x)$ is then introduced directly into the elastic stiffness $k$, that turns into a degrading stiffness through the following relation:

$$
k_{p}(\epsilon)=k\left[1-f_{\epsilon}(\epsilon) \rho_{p} g_{p}(x)\right],
$$

where $\rho_{p} \in[0,1]$ and $f_{\epsilon}(\epsilon)$ is defined as

$$
f_{\epsilon}(\epsilon)=1-e^{-\frac{1}{2}\left[\frac{\epsilon(t)}{\epsilon_{p}}\right]^{8}} .
$$

The parameter $\rho_{p}$ defines the amount of reduction with respect to the elastic stiffness $\left(\rho_{p}=0\right.$ means no pinching), while the function $f_{\epsilon}(\epsilon)$ has been introduced in order to make the pinching effect start at a certain activation energy, defined by the parameter $\epsilon_{p}$. The exponent in Equation (26) has been set to 8 in order to provide a fast activation of the pinching effect as soon as $\epsilon_{p}$ is reached. This high exponent does not produce singularities or numerical problems. Note that, regarding the simulation of the pinching phenomenon, the introduction of $f_{\epsilon}(\epsilon)$ represents the novelty of this work with respect to what was proposed by Pelliciari et al. (2018).

Figure 4a presents the effect of $\epsilon_{p}$ on the force-displacement response of the system. As $\epsilon_{p}$ increases, the activation of the pinching is delayed. The corresponding shape of the function $f_{\epsilon}(\epsilon)$ is shown in Figure $4 \mathrm{~b}$. It is clearly visible that when the system reaches the activation energy $\epsilon_{p}$ the 
Table 1. Summary of the parameters of the model and their roles.

\begin{tabular}{ccc}
\hline Parameter & Role \\
\hline 1 & $\alpha$ & ratio of linear to nonlinear response \\
2 & $k$ & elastic stiffness \\
3 & $\beta_{0}$ & hysteresis shape control \\
4 & $\eta_{0}$ & hysteresis shape control \\
5 & $n$ & hysteresis shape control \\
6 & $\rho_{x}$ & max. displacement degradation \\
7 & $\rho_{\epsilon}$ & energy degradation \\
8 & $\delta_{k}$ & stiffness degradation \\
9 & $\delta_{f}$ & strength degradation \\
10 & $\psi$ & stiffness degradation rate control \\
11 & $\sigma$ & pinching width \\
12 & $u$ & pinching slope \\
13 & $\rho_{p}$ & pinching severity \\
14 & $\epsilon_{p}$ & pinching activation energy \\
\hline
\end{tabular}

value of $f_{\epsilon}(\epsilon)$ goes rapidly from 0 to 1 , which causes the starting of the pinching effect. It should be noted that the case $\epsilon_{p}=0$ can not happen, because it would produce a singularity. However, since a real system never exhibits a pinching phenomenon that starts at the very beginning of the event, this case is not of interest.

The restoring force is then computed by substituting the elastic stiffness $k$ with the degrading stiffness $k_{p}(\epsilon)$, expressed by Equation (25), into Equation (2)

$$
F_{s}[x(t), z(t), t]=\alpha k_{p}(\epsilon) x(t)+(1-\alpha) k_{p}(\epsilon) z(t) .
$$

In conclusion, the parameters of the model are the following:

$$
\alpha, k, \beta_{0}, \eta_{0}, n, \rho_{x}, \rho_{\epsilon}, \delta_{k}, \delta_{f}, \psi, \sigma, u, \rho_{p}, \epsilon_{p}
$$

Table 1 summarizes the role that each parameter has on the definition of the hysteresis cycles. Although the model is composed of a significant number of parameters, each one has a specific physical meaning. Moreover, the pinching effect acts directly on the stiffness of the system through the parameters $\sigma, u, \rho_{p}$ and $\epsilon_{p}$, which can be easily identified and controlled. The relatively simple formulation of the degrading phenomena and the pinching effect brings benefit to the interpretation of the results and the use of the proposed model.

\section{Parameters optimization algorithm}

The values of the model parameters must be identified on the basis of experimental data. The aim is to find the optimal set of parameters, which provides the simulated response of the system that best fits the experimental one.

The set of parameters is collected into a vector $\boldsymbol{\theta}$, named parameter vector. The optimal parameter vector is denoted as $\boldsymbol{\theta}^{*}$ and it is the one that minimizes an objective function $(O F)$, which is a measure of discrepancy between numerical and experimental response. For the model proposed in this work, the parameters variation is constrained by defining the lower bound $\boldsymbol{\theta}^{l}$ and the upper bound $\boldsymbol{\theta}^{u}$.

Since the model must provide the best simulation of the cyclic behaviour of the system during the whole event, the $O F$ must be an integral measure over the whole set of experimental data. 
Therefore, the $O F$ is defined as

$$
O F(\boldsymbol{\theta})=\frac{\int_{x_{0}}^{x_{f}}\left|\left[F_{e}(x)-F_{s}(\boldsymbol{\theta}, x)\right] d x\right|}{\int_{x_{0}}^{x_{f}}\left|F_{e}(x) d x\right|},
$$

where $x_{i}$ and $x_{f}$ are the initial and final displacement records, $F_{e}(x)$ is the force derived from the experimental data and $F_{s}(\boldsymbol{\theta}, x)$ is the one simulated by the model. Hence, as expressed by Equation (28), the objective function is here defined as a normalized integral of the difference between experimental force and simulated force.

It is clear that the problem of minimization of the objective function described above is not simple. This is mainly due to the complexity of the differential equation that governs the hysteresis model (Equation (23)). Therefore, for such complex optimization problems, an evolutionary approach is usually adopted (Greco \& Vanzi, 2019; Quaranta, Marano, Greco, \& Monti, 2014).

Many identification of the parameters of structural models via a genetic algorithm (GA) can be found in literature. Marano, Quaranta, and Monti (2011) developed a modified real-coded genetic algorithm to identify the parameters of structural systems subjected to dynamic loads. Sengupta and Li (2013) used a GA to calibrate the parameters of a modified BWBN model applied to RC beam-column joints. Charalampakis and Koumousis (2008a) developed a hybrid evolutionary algorithm based on a GA, which was employed for the prediction of the behaviour of a steel cantilever beam. Sireteanu, Giuclea, and Mitu (2010) used a GA to identify the Bouc-Wen model parameters for elastomeric isolators and buckling restrained dissipative braces, while Ha, Kung, Fung, and Hsien (2006) performed the identification of a Bouc-Wen model via a real-coded genetic algorithm (RGA), for the case of piezoelectric actuators.

In view of this, the genetic algorithms represent suitable tools for complex identification problems. Thus, in this work, the optimization of the objective function is performed via a genetic algorithm.

\section{Application to reinforced concrete structural elements}

In the following, the model is applied to a RC bridge pier subjected to a cyclic test carried out in the lab of the Fuzhou University. Firstly, the experimental test is presented. Then, the parameters of the model are calibrated using the experimental data. Furthermore, comparisons with the results obtained with a model deprived first of the contribution of the maximum displacement $\left(\rho_{x}=0\right)$ and second of the dissipated energy $\left(\rho_{\epsilon}=0\right)$ are performed. This allows to assess the benefits of the introduction of both the energy and the maximum displacement into the damage index. Two further applications are then reported in order to ensure the reliability of the proposed model.

\subsection{Experimental test}

The identification of the model parameters is performed on the basis of the experimental data of a $\mathrm{RC}$ bridge pier. The experiment was carried out in the lab of the Fuzhou University (Xue et al., 2018). The pier considered is named R16-1B and it comes out from a repairing process of the original pier P16-1B, which was previously damaged by a cyclic test. The pier P16-1B had a circular cross section and it was made of Chinese concrete C30, steel HRB335E for the longitudinal reinforcement and steel R235 for the transversal reinforcement (JTG D62-2004, 2004). Its height and diameter were $1.17 \mathrm{~m}$ and $0.42 \mathrm{~m}$, respectively. The repairing consisted in the restoration of the damaged portion of the longitudinal steel reinforcement by means of turned rebar parts and substitution of the stirrups and the damaged concrete parts with HPFRC (High Performance Fiber Reinforced Concrete). A detailed description of the repairing process can be found in (Albanesi, Lavorato, Nuti, \& Santini, 2009; Lavorato \& Nuti, 2015; Lavorato, Nuti, Santini, Briseghella, \& Xue, 2015; Savino, Lanzoni, Tarantino, \& Viviani, 2018). 


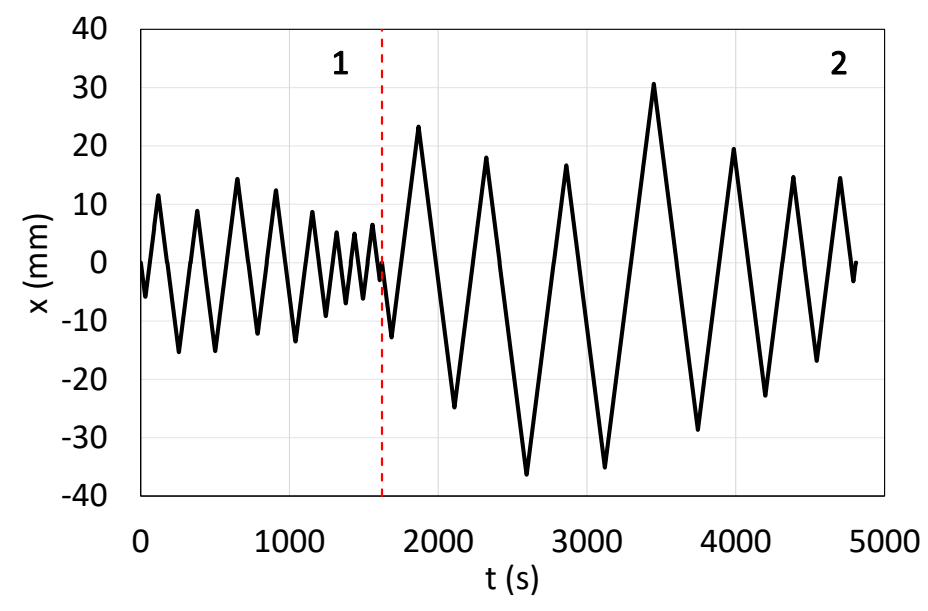

Figure 5. Displacement history applied at the top of the pier.

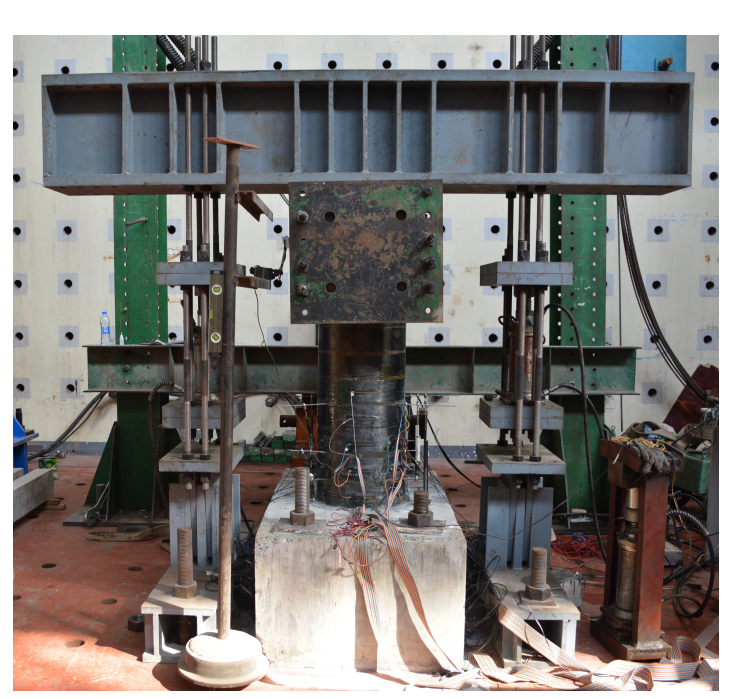

(a) Photograph of the test.

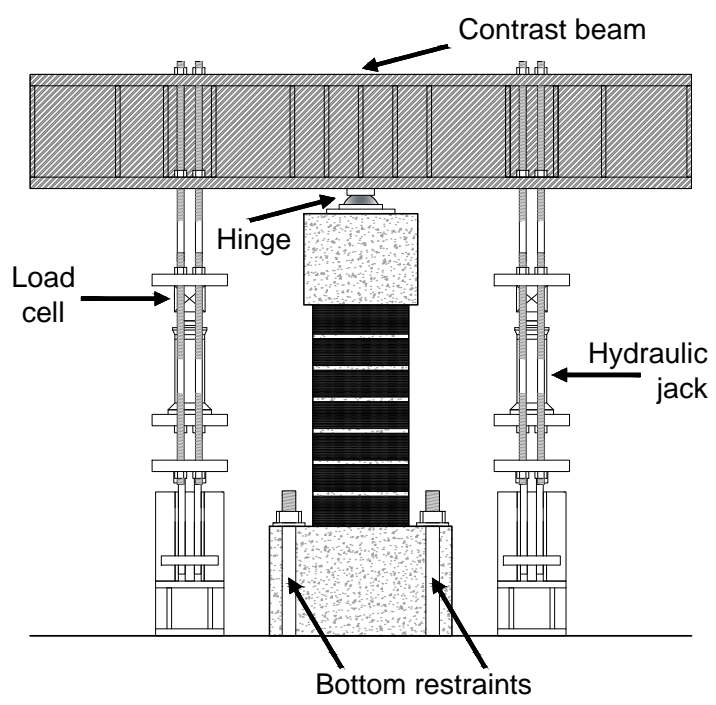

(b) Test setup.

Figure 6. Experimental test performed in the lab of the Fuzhou University.

The cyclic test, which was carried out firstly on the pier P16-1B and consequently on the pier R16-1B, consisted in the imposition of a displacement history at the top of the pier. As Figure 5 shows, the displacement history is composed of a first part and a second part. The first part corresponds to the response of the bridge column to the Tolmezzo accelerogram (Xue et al., 2018), referred to an earthquake occurred in Italy in 1976. The second part represents the response of the bridge column to the Tolmezzo accelerogram scaled to double. The Tolmezzo accelerogram has been selected because it is representative of a strong earthquake for the bridge analysed. The second part (scaled to double) has been added in order to further investigate the hysteresis behaviour of the pier when subject to severe damage.

The test setup is composed of a vertical load system and an horizontal actuator (Figures 6a and $6 \mathrm{~b}$ ). The vertical load system applies a constant load of $266 \mathrm{kN}$ on the top of the pier while the horizontal actuator impresses the displacement history. The constant vertical load represents the bridge deck load, properly scaled. As a result, the force-displacement curve is obtained. More details on the mechanical characteristics, the repairing process and the test setup are reported in (Xue et al., 2018). Figure 7 presents a schematic representation of the cross section of the pier R16-1B.

Similar experimental loading systems have been employed by several authors in order to obtain the behaviour of RC columns under cyclic lateral loading and a constant axial load, which 


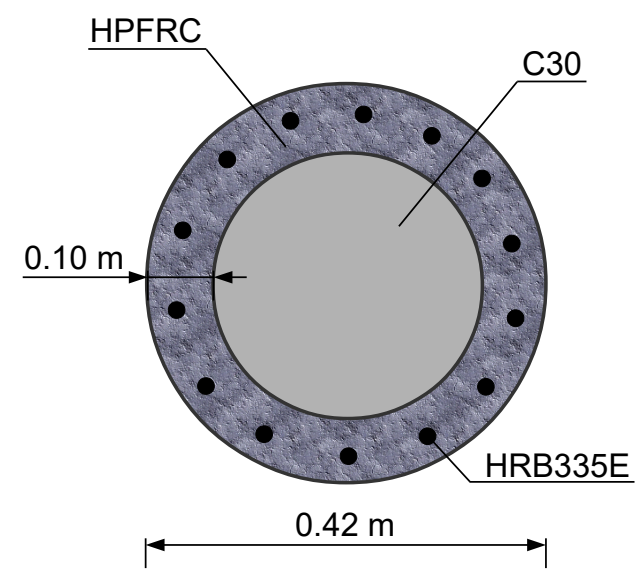

Figure 7. Cross section of the pier R16-1B.

simulates the dead load deriving from the superstructure (see, among the others, Colomb, Tobbi, Ferrier, \& Hamelin, 2008; He, Sneed, \& Belarbi, 2013; Sun, Wang, Du, \& Si, 2011).

\subsection{Calibration of the model parameters}

The experimental force-displacement data of the pier R16-1B are used to perform the identification of the optimal parameters of the model, which are gathered in the following parameter vector:

$$
\boldsymbol{\vartheta}=\left[\begin{array}{llllllllllllll}
\alpha & k & \beta_{0} & \eta_{0} & n & \rho_{x} & \rho_{\epsilon} & \delta_{k} & \delta_{f} & \psi & \sigma & u & \rho_{p} & \epsilon_{p}
\end{array}\right] .
$$

As pointed out by Pelliciari et al. (2018), a suitable value of $u$ for the description of the behaviour of $\mathrm{RC}$ piers is $u=4$. Thus, its value is fixed and the identification involves only the remaining 13 parameters. The values of ultimate displacement and horizontal normalized yielding force for the pier R16-1B are, respectively, $x_{u}=60 \mathrm{~mm}$ and $f_{y}=0.63 \mathrm{kN} / \mathrm{kg}$, considering the presence of a constant vertical load of $266 \mathrm{kN}$ applied on the top of the pier. The lower and upper bounds of the parameter vector are reported in Table 2 and the number of generations performed by the genetic algorithm is fixed to 100 .

The identification process is performed in the following three cases:

(1) the damage index includes both maximum displacement $x_{\max }(t)$ and dissipated energy $\epsilon(t)$, as expressed by Equation (9);

(2) the damage index does not include the maximum displacement $\left(\rho_{x}=0\right)$, therefore the degradation phenomena are only function of the dissipated energy $\epsilon(t)$;

(3) the damage index does not include the dissipated energy $\left(\rho_{\epsilon}=0\right)$, namely the degradation phenomena are only function of the maximum displacement $x_{\max }(t)$.

Table 3 lists the optimal parameters and the final $O F$ value for the three cases. As expected, the introduction of both maximum displacement and dissipated energy in the damage index (case 1) provides the best final simulated response, reaching an $O F$ around $9.5 \%$. Instead, the simulations in cases 2 and 3 provide an $O F$ that is approximately $5 \%$ and $1 \%$ higher, respectively.

Figures $8 \mathrm{a}, 8 \mathrm{~b}$ and $8 \mathrm{c}$ present the comparison between experimental data $\left(F_{\text {exp }}\right)$ and simulated result $\left(F_{s}\right)$ in terms of force-displacement curves in case 1, 2 and 3 , respectively. The simulation in case 1 (Figure 8a) shows a good fitting of the experimental hysteresis curve during the whole test. Instead, in case 2 (Figure $8 \mathrm{~b}$ ), it can be observed that the simulated behaviour is not accurate for the last hysteresis cycles, i.e. when the damage is severe. This is confirmed by the plot of experimental energy $\epsilon_{\exp }$ and simulated energy $\epsilon_{s}$ (Figure $9 \mathrm{~b}$ ), which shows that after a certain point of the test the simulated dissipation of energy is significantly lower than the experimental 
Table 2. Lower and upper boundaries of the parameters.

\begin{tabular}{cccc}
\hline Parameter & Unit of measure & Lower boundary & Upper boundary \\
\hline$\alpha$ & - & 0.03 & 0.1 \\
$k$ & $\mathrm{kN} / \mathrm{mm}$ & 35 & 60 \\
$\beta_{0}$ & - & 0 & 0.5 \\
$\eta_{0}$ & - & -1 & 1 \\
$n$ & - & 1 & 2 \\
$\delta_{k}$ & - & $1 \times 10^{-4}$ & $4 \times 10^{-4}$ \\
$\delta_{f}$ & - & $1 \times 10^{-5}$ & $2 \times 10^{-4}$ \\
$\psi$ & - & $4 \times 10^{-5}$ & $8 \times 10^{-5}$ \\
$\sigma$ & $\mathrm{mm}$ & 10 & 25 \\
$\rho_{p}$ & - & 0.1 & 0.5 \\
$\epsilon_{p}$ & $\mathrm{kN} \mathrm{mm} / \mathrm{kg}$ & 1 & 100 \\
$\rho_{x}$ & - & 0 & $3 \times 10^{4}$ \\
$\rho_{\epsilon}$ & - & 0 & $3 \times 10^{4}$ \\
\hline
\end{tabular}

Table 3. Best fitting parameters and $O F$ value for the pier R16-1B in case 1 (damage index involving both $x_{\max }(t)$ and $\epsilon(t))$, case $2\left(\rho_{x}=0\right)$ and case $3\left(\rho_{\epsilon}=0\right)$.

\begin{tabular}{ccccc}
\hline \multirow{2}{*}{ Parameter } & Unit of measure & \multicolumn{3}{c}{ Best fitting parameters } \\
& & Case 1 & Case 2 & Case 3 \\
\hline$\alpha$ & - & 0.0489 & 0.0869 & 0.0498 \\
$k$ & $\mathrm{kN} / \mathrm{mm}$ & 51.341 & 40.067 & 42.320 \\
$\beta_{0}$ & - & 0.214 & 0.0985 & 0.215 \\
$\eta_{0}$ & - & -0.275 & 0.188 & -0.338 \\
$n$ & - & 1.061 & 1.270 & 1.018 \\
$\delta_{k}$ & - & $2.796 \times 10^{-4}$ & $2.284 \times 10^{-4}$ & $2.709 \times 10^{-4}$ \\
$\delta_{f}$ & - & $3.790 \times 10^{-5}$ & $2.620 \times 10^{-5}$ & $2.686 \times 10^{-5}$ \\
$\psi$ & - & $6.896 \times 10^{-5}$ & $6.676 \times 10^{-5}$ & $7.667 \times 10^{-5}$ \\
$\sigma$ & $\mathrm{mm}$ & 16.032 & 12.734 & 19.709 \\
$\rho_{p}$ & - & 0.292 & 0.138 & 0.339 \\
$\epsilon_{p}$ & $\mathrm{kN} \mathrm{mm} / \mathrm{kg}$ & 43.220 & 35.673 & 47.716 \\
$\rho_{x}$ & - & $2.645 \times 10^{4}$ & 0 & $2.928 \times 10^{4}$ \\
$\rho_{\epsilon}$ & - & $1.526 \times 10^{3}$ & $1.492 \times 10^{4}$ & 0 \\
& & & & \\
\hline \multirow{2}{*}{$O F$} & & $9.53 \%$ & $14.94 \%$ & $10.60 \%$ \\
\hline
\end{tabular}




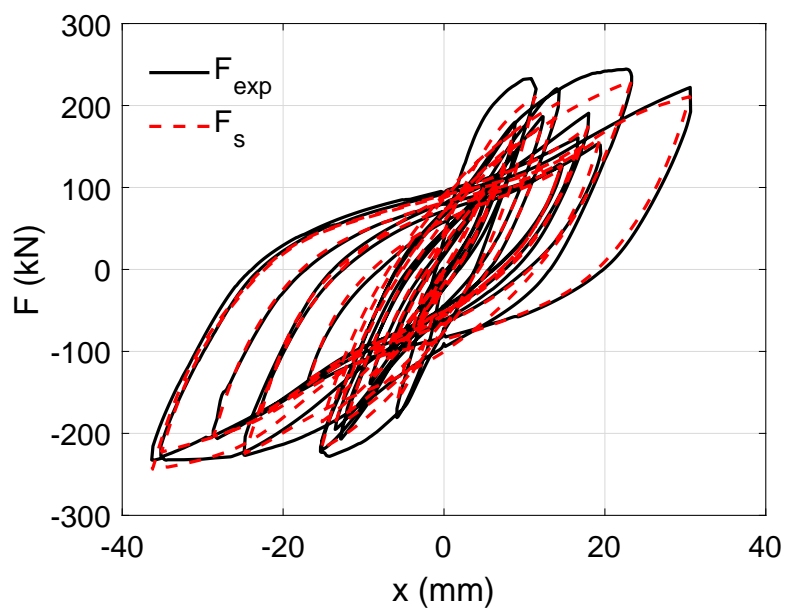

(a) Case 1 (damage index involving both maximum displacement and dissipated energy).

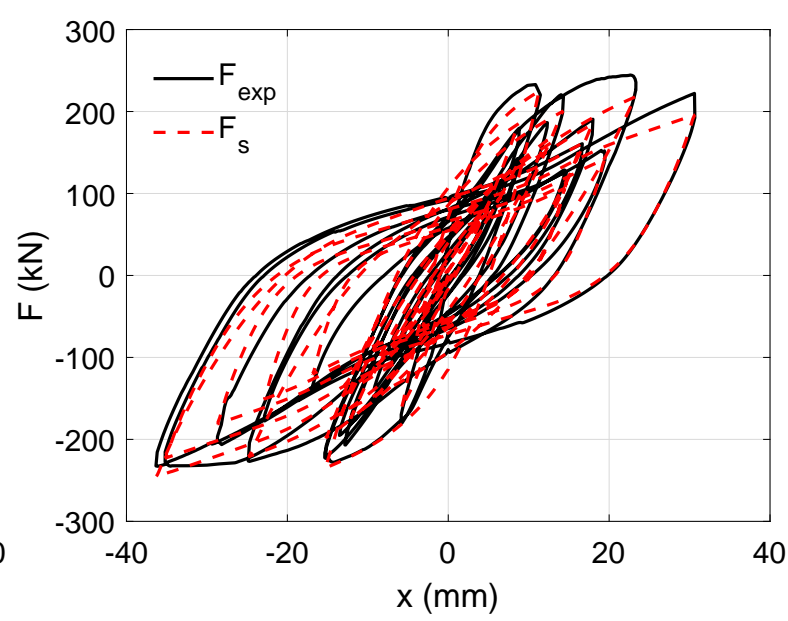

(b) Case $2\left(\rho_{x}=0\right)$.

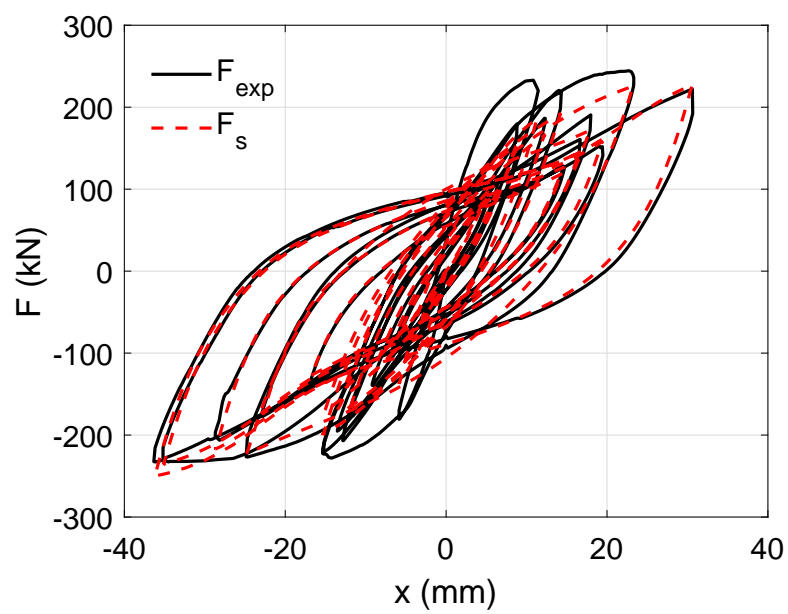

(c) Case $3\left(\rho_{\epsilon}=0\right)$.

Figure 8. Experimental $\left(F_{\text {exp }}\right)$ and simulated $\left(F_{s}\right)$ force-displacement curves for the pier R16-1B. 


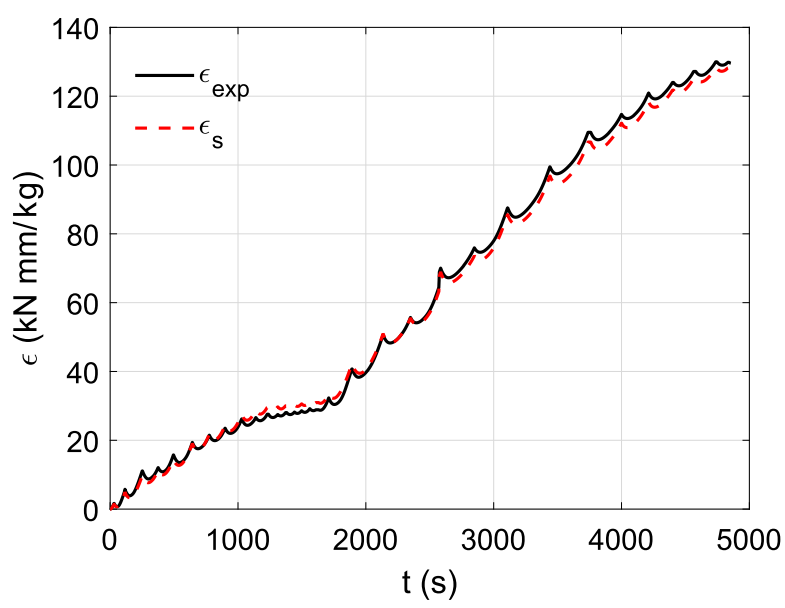

(a) Case 1 (damage index involving both maximum displacement and dissipated energy).

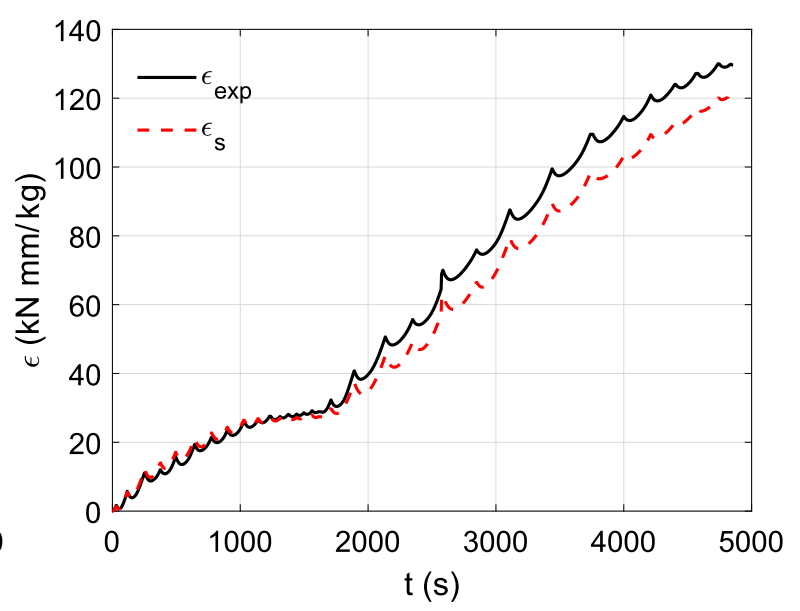

(b) Case $2\left(\rho_{x}=0\right)$.

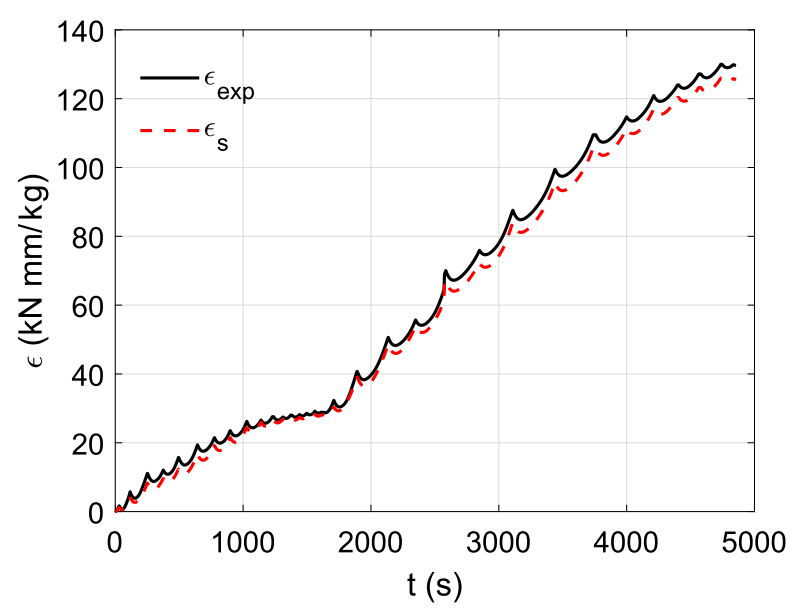

(c) Case $3\left(\rho_{\epsilon}=0\right)$.

Figure 9. Experimental $\left(\epsilon_{\text {exp }}\right)$ and simulated $\left(\epsilon_{s}\right)$ energy-time curves for the pier R16-1B.

one. It follows that the description of the damage as a function of the sole hysteretic energy is not satisfactory for the complex behaviour analysed here, while case 1 provides a more sound and reliable result (Figure 9a).

Turning to the force-displacement results in case 3 (Figures $8 \mathrm{c}$ and 9c), it is noted that the simulation fits quite well the experimental behaviour. Although, the first hysteresis cycles (i.e. when the degradation is still not severe) are not adequately described. The surprisingly low value of the $O F$ (see Table 3), almost as low as the simulation in case 1, is explained by the fact that the main discrepancy of the simulation is concentrated in the first part of the test, during which the restoring force of the system assumes relatively low values. Therefore, this discrepancy has a small influence on the magnitude of the $O F$, that is an integral measure over the whole event (Equation (28)). Indeed, the $O F$ alone would denote that the simulation in case 3 is nearly as accurate as that in case 1 . However, it follows from the analysis of the simulated response that its accuracy is significantly lower than in case 1.

Figure 10 shows the hysteretic response of the system divided into two parts. The dividing point is the instant of time when the displacement history switches from the first part to the second part (Figure 5). As already discussed, the simulation in case 1 provides a well fitting of the experimental behaviour throughout the event (Figures 10a and 10b). Instead, the simulated behaviour in case 2 is accurate in the first part, while there is a significant discrepancy in the second part (Figures 10c and 10d). Lastly, the simulation in case 3 is capable of describing the second part, whereas the first part is affected by a considerable error (Figures 10e and 10f).

In order to provide a comparison of the local accuracy of each simulation, the following relative 


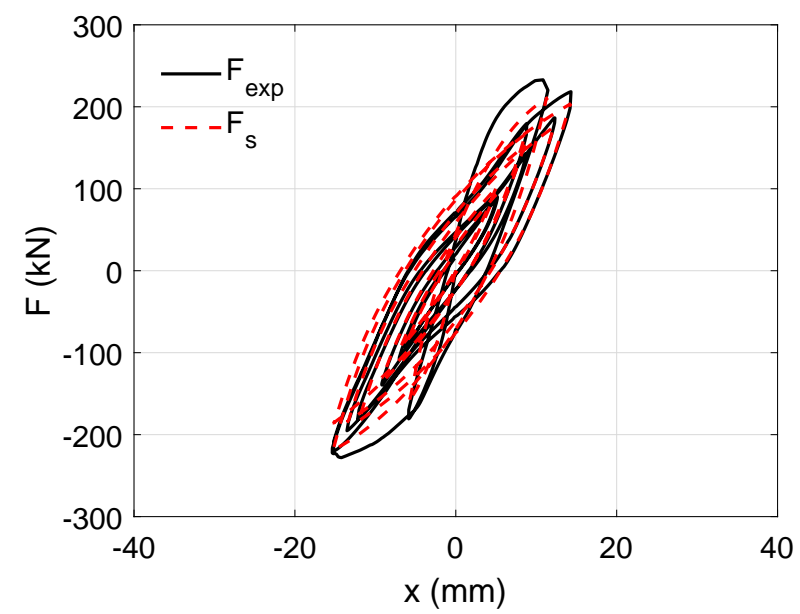

(a) Case 1: first part of displacement history.

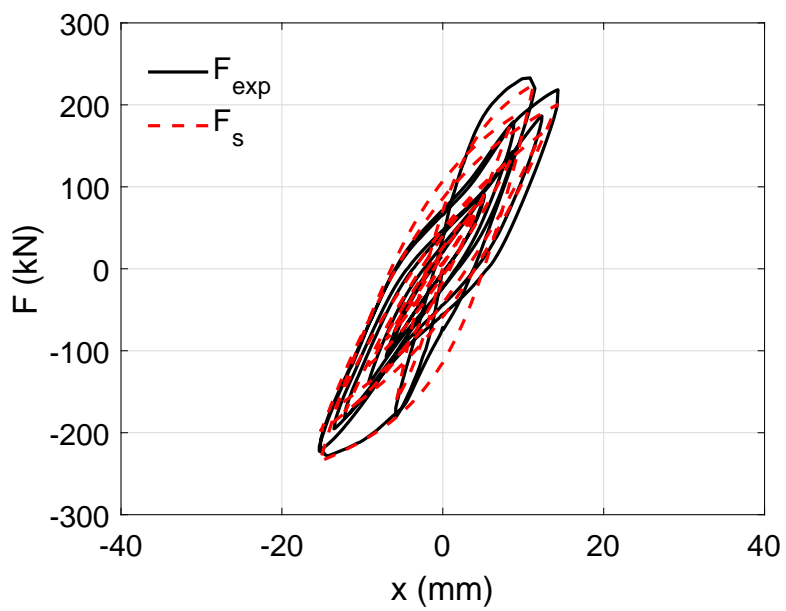

(c) Case 2: first part of displacement history.

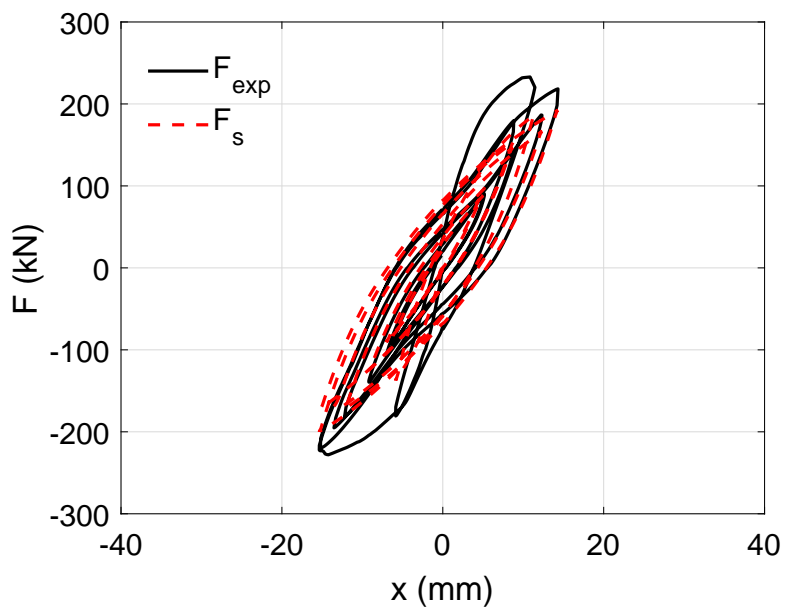

(e) Case 3: first part of displacement history.

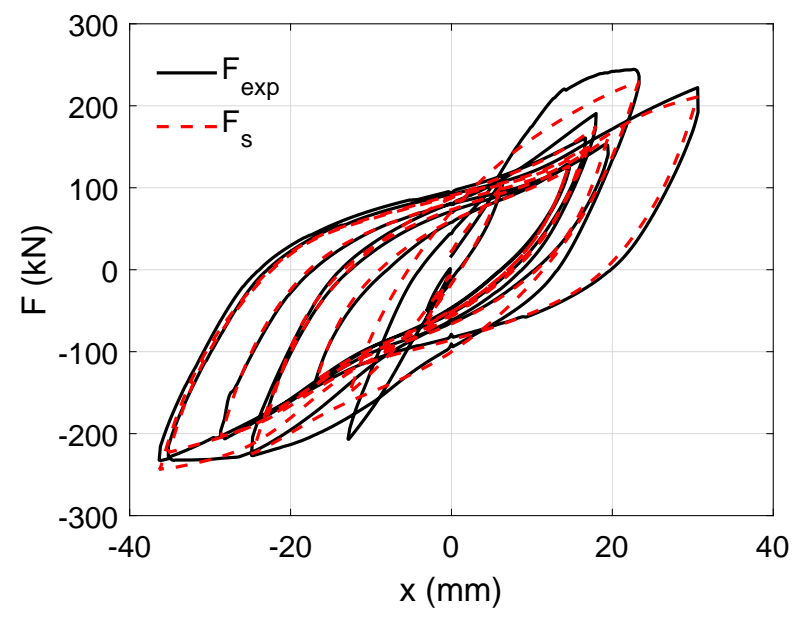

(b) Case 1: second part of displacement history.

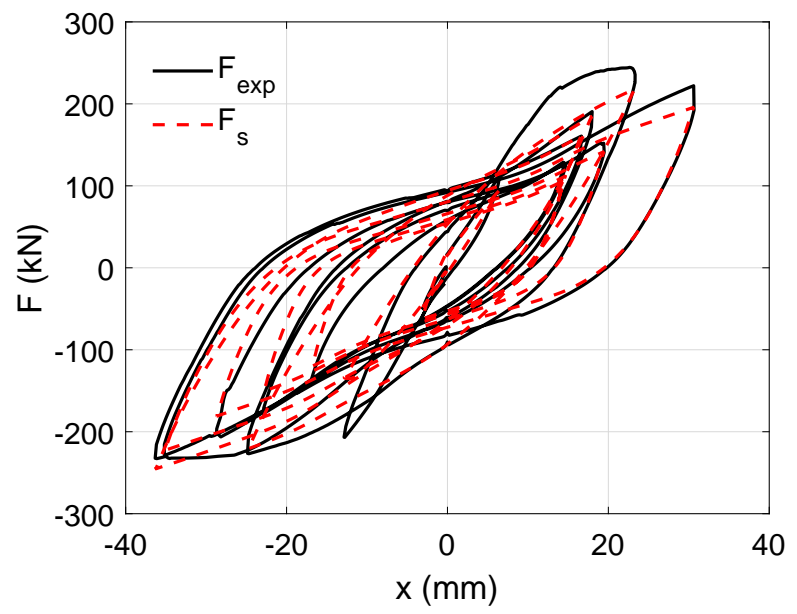

(d) Case 2: second part of displacement history.

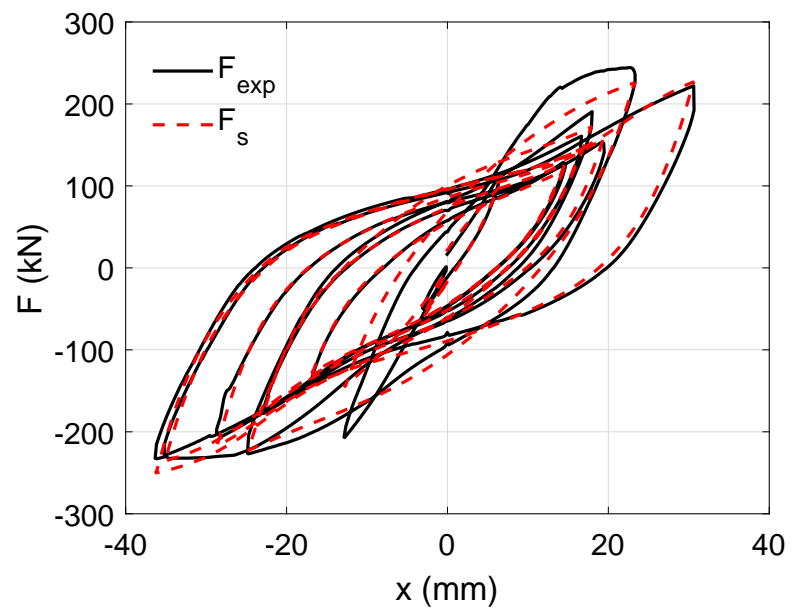

(f) Case 3: second part of displacement history.

Figure 10. Experimental $\left(F_{\text {exp }}\right)$ and simulated $\left(F_{s}\right)$ response of the pier R16-1B divided into two parts. 


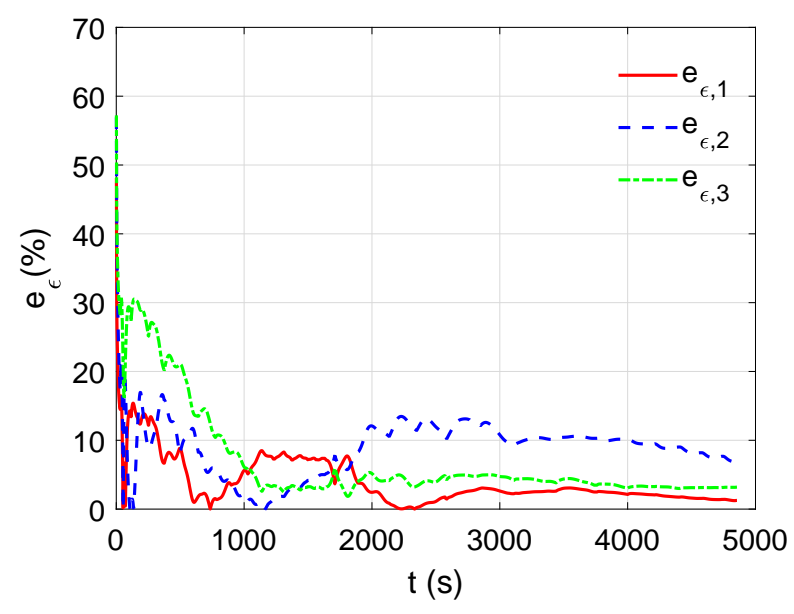

Figure 11. Relative error $e_{r}$ in terms of dissipated energy committed in case $1\left(e_{\epsilon, 1}\right)$, case $2\left(e_{\epsilon, 2}\right)$ and case $3\left(e_{\epsilon, 3}\right)$.

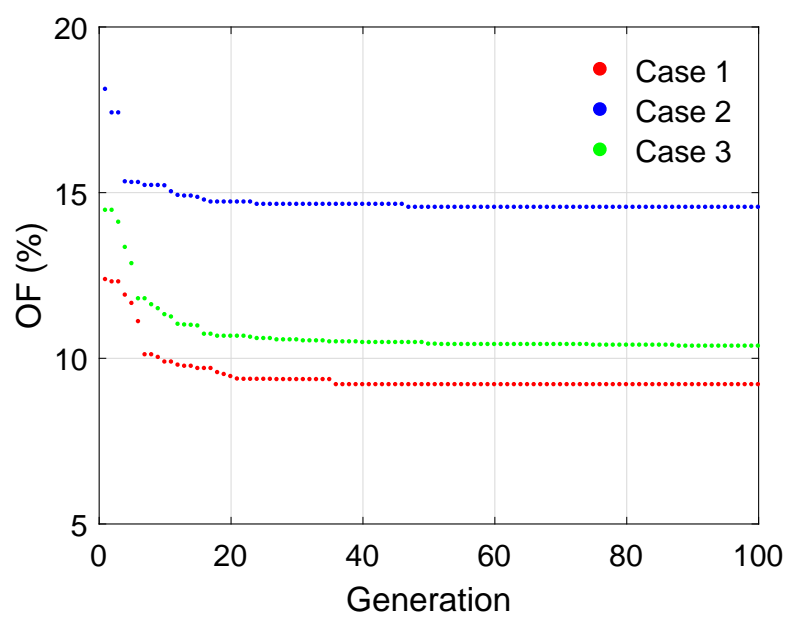

Figure 12. $O F$ values along with the generations during the identification process for the pier R16-1B in case 1 , case 2 and case 3 .

error in terms of energy is computed:

$$
e_{\epsilon}(\boldsymbol{\theta}, t)=\frac{\left|\epsilon_{\exp }(t)-\epsilon_{s}(\boldsymbol{\theta}, t)\right|}{\epsilon_{\exp }(t)} .
$$

The errors made in case $1\left(e_{\epsilon, 1}\right)$, case $2\left(e_{\epsilon, 2}\right)$ and case $3\left(e_{\epsilon, 3}\right)$ are displayed in Figure 11. The plot confirms all the above observations. In fact, the error in case 3 is higher during the first part of the test, while the error in case 2 is higher during the second part of the test. The simulation in case 1 provides the lowest local error during most of the test. Therefore, it is concluded that the incorporation of both maximum displacement and energy in the damage index brings benefits to the capability of the model to simulate the hysteresis behaviour of the system.

Finally, Figure 12 shows the value of the objective function with the progress of the generations in case 1, 2 and 3. For each case, the simulation has already reached a good result after 20 generations. This indicates that the genetic algorithm does not struggle too much to find the optimal solution, thanks to the relatively simple formulation of the model.

\subsection{Further applications of the proposed model}

In order to guarantee the reliability of the hysteresis model presented in this paper, two applications on rectangular $\mathrm{RC}$ columns are now presented. The experimental data were obtained 


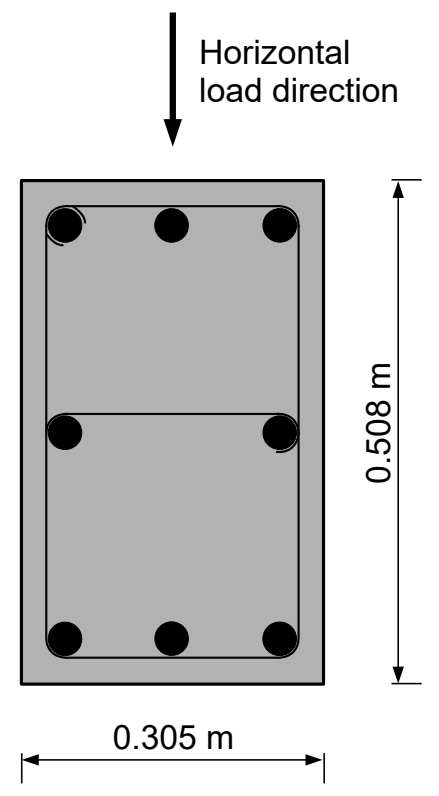

(a) Column ORC1.

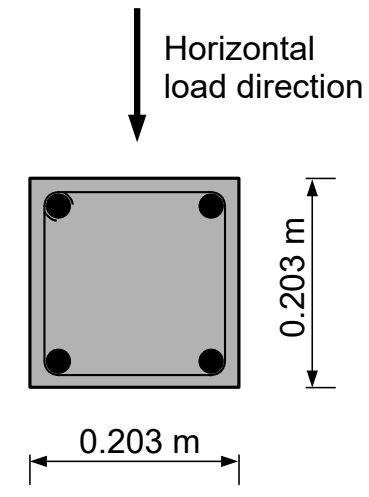

(b) Column C5-00.

Figure 13. Cross sections of the two RC columns analysed.

from the PEER database for reinforced concrete elements (Berry, Parrish, \& Eberhard, 2004, https://nisee.berkeley.edu/spd). Since the purpose of the following applications is to further investigate the reliability of the proposed model, the two cases with $\rho_{x}=0$ and $\rho_{\epsilon}=0$ are not considered. The previous application already demonstrated that the introduction of a damage index containing both $\rho_{x}$ and $\rho_{\epsilon}$ allows reaching a higher accuracy.

The first application regards the column ORC1, whose cyclic response was experimentally investigated by Aboutaha and Machado (1999). The column ORC1 was composed of a concrete with strength $83 \mathrm{MPa}$, reinforced with eight $25 \mathrm{~mm}$ and grade $414 \mathrm{MPa}$ longitudinal reinforcing bars. The size of the cross section was $305 \times 508 \mathrm{~mm}$, while the height of the column was 1.829 $\mathrm{m}$. The transversal reinforcement was formed by 4-legged shear stirrups with a diameter of 10 $\mathrm{mm}$ and steel grade $414 \mathrm{MPa}$, spaced at $75 \mathrm{~mm}$ in the potential plastic hinge region and $150 \mathrm{~mm}$ outside that region. The ultimate displacement and horizontal normalized yielding force for the specimen ORC1 are $x_{u}=109.74 \mathrm{~mm}$ and $f_{y}=0.29 \mathrm{kN} / \mathrm{kg}$.

The second application concerns the column C5-00, which was tested by Matamoros (2000). The column C5-00 was composed of a concrete with strength $37.9 \mathrm{MPa}$. The rectangular cross section had dimensions of $203 \times 203 \mathrm{~mm}$ and it was longitudinally reinforced with four bars with diameter $15.9 \mathrm{~mm}$ and yielding strength $572.3 \mathrm{MPa}$. The height of the column was $0.61 \mathrm{~m}$ and the transversal reinforcement was performed by introducing 2-legged shear stirrups with a diameter of $9.5 \mathrm{~mm}$ and yielding stress $513.7 \mathrm{MPa}$, at a spacing of $76.2 \mathrm{~mm}$. The column C5-00 has an ultimate displacement $x_{u}=50.8 \mathrm{~mm}$ and an horizontal normalized yielding force $f_{y}=0.84$ $\mathrm{kN} / \mathrm{kg}$.

Both the columns ORC1 and C5-00 were tested under the imposition of a cyclic horizontal displacement at the top of the system, while no axial load was applied. The section at the bottom of the columns was fixed, thus the test configuration was a cantilever. Figures 13a and 13b show a schematic representation of the cross sections of the columns ORC1 and C5-00, respectively.

The experimental force-displacement hysteresis curves of the two columns are used to perform the identification of the optimal parameters of the model for both of them. Once again, the parameter $u$ is fixed to the value 4 (see Section 4.2) and the number of generations performed by the genetic algorithm is set to 100 . The lower and upper bounds of the parameters are the same as the ones listed in Table 2, with the exceptions of the ranges of $k, \sigma$ and $\epsilon_{p}$. These three parameters are not dimensionless, therefore their ranges must be adjusted basing on the particular system 
Table 4. Best fitting parameters and $O F$ value for the columns ORC1 and C5-00.

\begin{tabular}{cccc}
\hline \multirow{2}{*}{ Parameter } & Unit of measure & \multicolumn{2}{c}{ Best fitting parameters } \\
& & ORC1 & C5-00 \\
\hline$\alpha$ & - & 0.0474 & 0.0350 \\
$k$ & $\mathrm{kN} / \mathrm{mm}$ & 21.013 & 9.314 \\
$\beta_{0}$ & - & 0.0764 & 0.102 \\
$\eta_{0}$ & - & -0.588 & -0.790 \\
$n$ & - & 1.046 & 1.124 \\
$\delta_{k}$ & - & $2.494 \times 10^{-4}$ & $3.250 \times 10^{-4}$ \\
$\delta_{f}$ & - & $4.076 \times 10^{-5}$ & $1.641 \times 10^{-4}$ \\
$\psi$ & - & $4.576 \times 10^{-5}$ & $4.576 \times 10^{-5}$ \\
$\sigma$ & $\mathrm{mm}$ & 73.401 & 10.677 \\
$\rho_{p}$ & - & 0.103 & 0.221 \\
$\epsilon_{p}$ & $\mathrm{kN} \mathrm{mm/kg}$ & 339.914 & 35.991 \\
$\rho_{x}$ & - & $2.765 \times 10^{4}$ & $1.271 \times 10^{4}$ \\
$\rho_{\epsilon}$ & - & 495.137 & 531.500 \\
& & & \\
\hline \multirow{2}{*}{$O F$} & & $11.39 \%$ & $9.92 \%$ \\
\hline
\end{tabular}

being examined. For this reason, both for the column ORC1 and $\mathrm{C} 5-00$, their ranges have been modified before running the optimization process.

Table 4 lists the optimal parameters and the final OF value for the columns ORC1 and C500. Figures $14 \mathrm{a}$ and $14 \mathrm{~b}$ show the experimental data and the simulated force-displacement curves for the columns ORC1 and C5-00, respectively. It can be observed that, for both systems, the hysteresis behaviour simulated by the model is well fitting the experimental one. In fact, the error in terms of $O F$ is around $10 \%$ in both cases.

It is worth noting that the pronounced strength degradation and pinching effect of the column C5-00 with respect to the column ORC1 is directly reflected on the values of the parameters $\delta_{f}$ and $\rho_{p}$. In fact, as reported in Table 4, the optimal values of $\delta_{f}$ and $\rho_{p}$ for the column C5-00 are sensibly higher than those for the column ORC1.

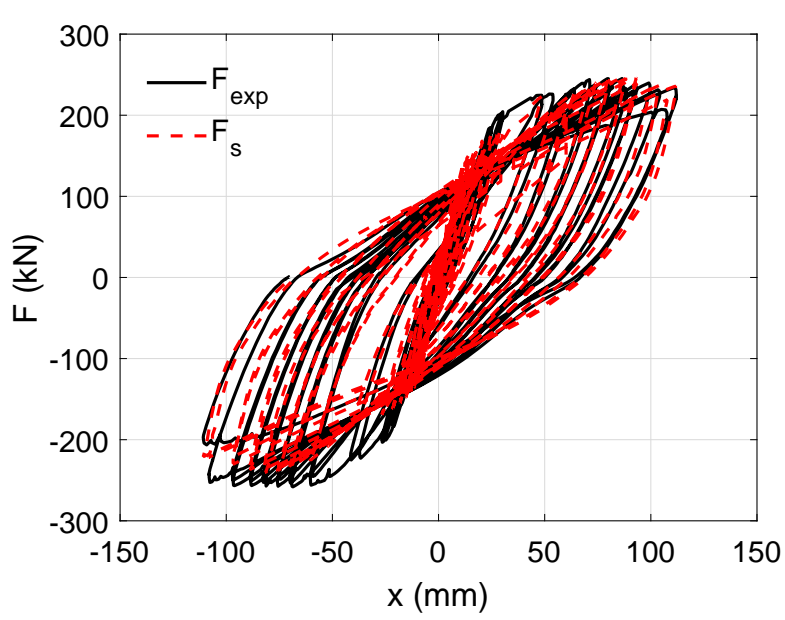

(a) Column ORC1.

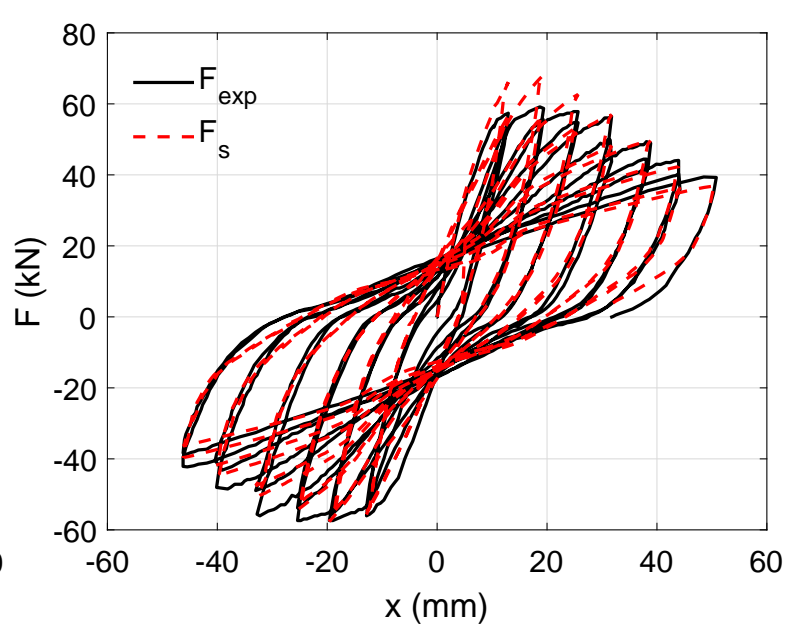

(b) Column C5-00.

Figure 14. Experimental $\left(F_{\text {exp }}\right)$ and simulated $\left(F_{s}\right)$ force-displacement curves for the RC columns analysed. 


\section{Conclusions}

This paper presents a smooth hysteresis model for RC structural elements with damage and pinching effects. Its formulation is based on the differential equation of the Bouc-Wen model, which has been extensively used for the description of the hysteresis of various mechanical systems.

The deterioration of the mechanical characteristics is introduced through a damage index, that is defined as a linear combination of dissipated energy and maximum displacement. Stiffness and strength degradation are mathematically described as decoupled phenomena using negative exponential laws. Furthermore, the pinching effect is introduced in a similar way as what was done by Marano et al. (2017) and Pelliciari et al. (2018). However, the model proposed in the abovementioned works involves a pinching phenomenon that starts at the beginning of the event, affecting also the very first hysteresis cycles. In the present paper, this physical inconsistency is resolved by including an activation energy for the pinching effect.

The model is applied to the hysteresis loops of a RC bridge pier tested in the lab of the Fuzhou University. The following three cases are analysed: damage index involving both dissipated energy and maximum displacement, damage index involving only dissipated energy and damage index involving only maximum displacement. The results show that the description of the damage as a function of both dissipated energy and maximum displacement produces a simulation that well fits the experimental hysteresis curve during the whole test. Instead, modeling the damage as a function of the sole dissipated energy leads to an inadequate simulation of the behaviour of the pier when the damage is severe. On the contrary, a description of the degradation considering only the maximum displacement is not accurate during the first part of the test, where the damage is still slight.

Two further applications on rectangular RC columns are also presented, with the aim of ensuring the reliability of the proposed model. The results demonstrate that the model is capable of reproducing the complex hysteresis behaviour of the structural elements analysed, that involves stiffness degradation, strength degradation and pinching effect. The simple formulation of the model allows to perform the identification of the best fitting parameters with a small computational burden. Moreover, each parameter has a direct physical meaning, which helps in the interpretation of the results.

The hysteresis model presented in this work is oriented towards practical engineering applications, with a view to providing an instrument that can be easily managed and applied to simulate the hysteresis of RC structural elements. Although the applications presented provided accurate results, the general applicability of the model has to be further investigated. In order to verify this, future developments will involve simulations on other types of RC elements, such as beam-column joints, frames, walls, etc.

\section{Acknowledgments}

The authors acknowledge the Sustainable and Innovative Bridge Engineering Research Center (SIBERC) of the College of Civil Engineering of the Fuzhou University (Fuzhou, China) and the PRISMA lab of the Department of Architecture of the Roma Tre University (Rome, Italy).

\section{Disclosure statement}

No potential conflict of interest was reported by the authors.

\section{Fundings}

This work was supported by the National Natural Science Foundation of China [grant number 51778148]; the Recruitment Program of Global Experts Foundation [grant number TM2012- 
27]; and the Laboratories University Network of Seismic Engineering (ReLUIS) [research project ReLUIS/DPC 2016-2018].

\section{References}

Aboutaha, R. S., \& Machado, R. I. (1999). Seismic resistance of steel-tubed high-strength reinforcedconcrete columns. Journal of Structural Engineering, 125(5), 485-494.

Albanesi, T., Lavorato, D., Nuti, C., \& Santini, S. (2009). Experimental program for pseudodynamic tests on repaired and retrofitted bridge piers. European Journal of Environmental and Civil Engineering, 13(6), 671-683.

Baber, T. T., \& Noori, M. N. (1985). Random vibration of degrading, pinching systems. Journal of Engineering Mechanics, $111(8), 1010-1026$.

Baber, T. T., \& Wen, Y. K. (1980). Stochastic equivalent linearization for hysteretic, degrading, multistory structures. University of Illinois Engineering Experiment Station, College of Engineering, University of Illinois at Urbana-Champaign.

Banon, H., \& Veneziano, D. (1982). Seismic safety of reinforced concrete members and structures. Earthquake Engineering \& Structural Dynamics, 10(2), 179-193.

Bassam, A., Iranmanesh, A., \& Ansari, F. (2011). A simple quantitative approach for post earthquake damage assessment of flexure dominant reinforced concrete bridges. Engineering Structures, 33(12), $3218-3225$.

Bažant, Z. P., Pan, J., \& Pijaudier-Cabot, G. (1987). Softening in reinforced concrete beams and frames. Journal of Structural Engineering, 113(12), 2333-2347.

Berry, M., Parrish, M., \& Eberhard, M. (2004). Peer structural performance database, user's manual (version 1.0). University of California, Berkeley.

Bouc, R. (1971). A mathematical model for hysteresis. Acta Acustica united with Acustica, 24 (1), 16-25.

Charalampakis, A. E., \& Dimou, C. K. (2010). Identification of Bouc-Wen hysteretic systems using particle swarm optimization. Computers \& Structures, 88(21-22), 1197-1205.

Charalampakis, A. E., \& Koumousis, V. K. (2008a). Identification of Bouc-Wen hysteretic systems by a hybrid evolutionary algorithm. Journal of Sound and Vibration, 314(3-5), 571-585.

Charalampakis, A. E., \& Koumousis, V. K. (2008b). On the response and dissipated energy of Bouc-Wen hysteretic model. Journal of Sound and Vibration, 309(3-5), 887-895.

Colomb, F., Tobbi, H., Ferrier, E., \& Hamelin, P. (2008). Seismic retrofit of reinforced concrete short columns by CFRP materials. Composite Structures, 82(4), 475-487.

Cunha, Á. A. M. F. (1994). The role of the stochastic equivalent linearization method in the analysis of the non-linear seismic response of building structures. Earthquake Engineering 83 Structural Dynamics, 23(8), 837-857.

Domaneschi, M. (2012). Simulation of controlled hysteresis by the semi-active Bouc-Wen model. Computers \&3 Structures, 106, 245-257.

Elenas, A. (2000). Correlation between seismic acceleration parameters and overall structural damage indices of buildings. Soil Dynamics and Earthquake Engineering, 20(1-4), 93-100.

Erlicher, S., \& Bursi, O. S. (2008). Bouc-Wen-type models with stiffness degradation: thermodynamic analysis and applications. Journal of Engineering Mechanics, 134 (10), 843-855.

Foliente, G. C. (1995). Hysteresis modeling of wood joints and structural systems. Journal of Structural Engineering, 121(6), 1013-1022.

Greco, R., \& Marano, G. C. (2015). Inelastic seismic spectra including a damage criterion: A stochastic approach. Soil Dynamics and Earthquake Engineering, 70, 75-79.

Greco, R., \& Vanzi, I. (2019). New few parameters differential evolution algorithm with application to structural identification. Journal of Traffic and Transportation Engineering (English Edition), 6(1), $1-14$.

Ha, J. L., Kung, Y. S., Fung, R. F., \& Hsien, S. C. (2006). A comparison of fitness functions for the identification of a piezoelectric hysteretic actuator based on the real-coded genetic algorithm. Sensors and Actuators A: Physical, 132(2), 643-650.

He, R., Sneed, L. H., \& Belarbi, A. (2013). Rapid repair of severely damaged RC columns with different damage conditions: An experimental study. International Journal of Concrete Structures and Materials, 7(1), 35-50.

Ismail, M., Ikhouane, F., \& Rodellar, J. (2009). The hysteresis Bouc-Wen model, a survey. Archives of Computational Methods in Engineering, 16(2), 161-188. 
Ismail, M., Rodellar, J., \& Ikhouane, F. (2010). An innovative isolation device for aseismic design. Engineering Structures, 32(4), 1168-1183.

JTG D62-2004. (2004). Code for design of highway reinforced concrete and prestressed concrete bridges and culverts. Beijing: China Communications Press.

Kashani, M. M., Salami, M. R., Goda, K., \& Alexander, N. A. (2018). Non-linear flexural behaviour of RC columns including bar buckling and fatigue degradation. Magazine of Concrete Research, $70(5)$, 231-247.

Kim, S. Y., \& Lee, C. H. (2019). Description of asymmetric hysteretic behavior based on the Bouc-Wen model and piecewise linear strength-degradation functions. Engineering Structures, 181, 181-191.

Kottari, A. K., Charalampakis, A. E., \& Koumousis, V. K. (2014). A consistent degrading Bouc-Wen model. Engineering Structures, 60, 235-240.

Kunnath, S. K., Mander, J. B., \& Fang, L. (1997). Parameter identification for degrading and pinched hysteretic structural concrete systems. Engineering Structures, 19(3), 224-232.

Kunnath, S. K., Reinhorn, A. M., \& Park, Y. J. (1990). Analytical modeling of inelastic seismic response of R/C structures. Journal of Structural Engineering, 116(4), 996-1017.

Lanzoni, L., \& Tarantino, A. M. (2014). Damaged hyperelastic membranes. International Journal of Non-Linear Mechanics, 60, 9-22.

Lanzoni, L., \& Tarantino, A. M. (2015). Equilibrium configurations and stability of a damaged body under uniaxial tractions. Zeitschrift für angewandte Mathematik und Physik, 66(1), 171-190.

Lanzoni, L., \& Tarantino, A. M. (2016). A simple nonlinear model to simulate the localized necking and neck propagation. International Journal of Non-Linear Mechanics, 84, 94-104.

Lavorato, D., \& Nuti, C. (2015). Pseudo-dynamic tests on reinforced concrete bridges repaired and retrofitted after seismic damage. Engineering Structures, 94, 96-112.

Lavorato, D., Nuti, C., Santini, S., Briseghella, B., \& Xue, J. (2015). A repair and retrofitting intervention to improve plastic dissipation and shear strength of chinese RC bridges. In IABSE Symposium Report (Vol. 105, pp. 1-6).

Lee, C. S., \& Han, S. W. (2018). Computationally effective and accurate simulation of cyclic behaviour of old reinforced concrete columns. Engineering Structures, 173, 892-907.

Liu, T., Zordan, T., Zhang, Q., \& Briseghella, B. (2015). Equivalent viscous damping of bilinear hysteretic oscillators. Journal of Structural Engineering, $141(11), 06015002$.

Loh, C. H., Mao, C. H., Huang, J. R., \& Pan, T. C. (2011). System identification and damage evaluation of degrading hysteresis of reinforced concrete frames. Earthquake Engineering \& Structural Dynamics, 40(6), 623-640.

Ma, F., Zhang, H., Bockstedte, A., Foliente, G. C., \& Paevere, P. (2004). Parameter analysis of the differential model of hysteresis. Journal of Applied Mechanics, 71(3), 342-349.

Manzoori, A., \& Toopchi-Nezhad, H. (2017). Application of an extended Bouc-Wen model in seismic response prediction of unbonded fiber-reinforced isolators. Journal of Earthquake Engineering, 21(1), 87-104.

Marano, G. C., \& Greco, R. (2006). Damage and ductility demand spectra assessment of hysteretic degrading systems subject to stochastic seismic loads. Journal of Earthquake Engineering, 10(05), 615-640.

Marano, G. C., Pelliciari, M., Cuoghi, T., Briseghella, B., Lavorato, D., \& Tarantino, A. M. (2017). Degrading Bouc-Wen model parameters identification under cyclic load. International Journal of Geotechnical Earthquake Engineering, 8(2), 60-81.

Marano, G. C., Quaranta, G., \& Monti, G. (2011). Modified genetic algorithm for the dynamic identification of structural systems using incomplete measurements. Computer-Aided Civil and Infrastructure Engineering, 26(2), 92-110.

Markou, A. A., \& Manolis, G. D. (2016). Mechanical formulations for bilinear and trilinear hysteretic models used in base isolators. Bulletin of Earthquake Engineering, 14(12), 3591-3611.

Matamoros, A. B. (2000). Study of drift limits for high-strength concrete columns. Department of Civil Engineering, University of Illinois at Urbana-Champaign.

Mazars, J. (1986). A description of micro-and macroscale damage of concrete structures. Engineering Fracture Mechanics, 25(5-6), 729-737.

Miehe, C. (1995). Discontinuous and continuous damage evolution in Ogden-type large-strain elastic materials. European Journal of Mechanics - A/Solids, 14(5), 697-720.

Ortiz, G. A., Alvarez, D. A., \& Bedoya-RuíZ, D. (2013). Identification of Bouc-Wen type models using multi-objective optimization algorithms. Computers \& Structures, 114, 121-132.

Park, Y. J., \& Ang, A. H. S. (1985). Mechanistic seismic damage model for reinforced concrete. Journal 
of Structural Engineering, $111(4), 722-739$.

Pelliciari, M., Marano, G. C., Cuoghi, T., Briseghella, B., Lavorato, D., \& Tarantino, A. M. (2018). Parameter identification of degrading and pinched hysteretic systems using a modified Bouc-Wen model. Structure and Infrastructure Engineering, 14(12), 1573-1585.

Quaranta, G., Marano, G. C., Greco, R., \& Monti, G. (2014). Parametric identification of seismic isolators using differential evolution and particle swarm optimization. Applied Soft Computing, 22, $458-464$.

Ray, T., Sarlis, A. A., Reinhorn, A. M., \& Constantinou, M. C. (2013). Hysteretic models for sliding bearings with varying frictional force. Earthquake Engineering 6 Structural Dynamics, 42(15), 23412360 .

Roufaiel, M. S. L., \& Meyer, C. (1987). Analytical modeling of hysteretic behavior of R/C frames. Journal of Structural Engineering, 113(3), 429-444.

Savino, V., Lanzoni, L., Tarantino, A. M., \& Viviani, M. (2018). Simple and effective models to predict the compressive and tensile strength of HPFRC as the steel fiber content and type changes. Composites Part B: Engineering, 137, 153-162.

Sengupta, P., \& Li, B. (2013). Modified Bouc-Wen model for hysteresis behavior of RC beam-column joints with limited transverse reinforcement. Engineering Structures, 46, 392-406.

Singhal, A., \& Kiremidjian, A. S. (1996). Method for probabilistic evaluation of seismic structural damage. Journal of Structural Engineering, 122(12), 1459-1467.

Sireteanu, T., Giuclea, M., \& Mitu, A. M. (2010). Identification of an extended Bouc-Wen model with application to seismic protection through hysteretic devices. Computational Mechanics, 45(5), 431-441.

Sivaselvan, M. V., \& Reinhorn, A. M. (2000). Hysteretic models for deteriorating inelastic structures. Journal of Engineering Mechanics, 126(6), 633-640.

Sues, R. H., Mau, S. T., \& Wen, Y. K. (1988). Systems identification of degrading hysteretic restoring forces. Journal of Engineering Mechanics, 114(5), 833-846.

Sun, Z., Wang, D., Du, X., \& Si, B. (2011). Rapid repair of severely earthquake-damaged bridge piers with flexural-shear failure mode. Earthquake Engineering and Engineering Vibration, 10(4), 553-567.

Tarantino, A. M. (2014). Equilibrium paths of a hyperelastic body under progressive damage. Journal of Elasticity, $114(2), 225-250$.

Wang, X., Lu, X., \& Ye, L. (2007). Numerical simulation for the hysteresis behavior of RC columns under cyclic loads. Journal of Engineering Mechanics, 12, 017.

Wen, Y. K. (1976). Method for random vibration of hysteretic systems. Journal of Engineering Mechanics, 102(2), 249-263.

Xue, J., Lavorato, D., Bergami, A., Nuti, C., Briseghella, B., Marano, G., .. S Santini, S. (2018). Severely damaged reinforced concrete circular columns repaired by turned steel rebar and high-performance concrete jacketing with steel or polymer fibers. Applied Sciences, 8(9), 1671.

Ye, M., \& Wang, X. (2007). Parameter estimation of the Bouc-Wen hysteresis model using particle swarm optimization. Smart Materials and Structures, 16(6), 2341.

Zhu, W., \& Rui, X. T. (2016). Hysteresis modeling and displacement control of piezoelectric actuators with the frequency-dependent behavior using a generalized Bouc-Wen model. Precision Engineering, 43, 299-307. 